\title{
Variable Faint Optical Sources Discovered by Comparing the POSS and SDSS Catalogs
}

\section{Citation}

Sesar, Branimir, Domjan Svilkovic, Zeljko Ivezic, Robert H. Lupton, Jeffrey A. Munn, Douglas Finkbeiner, William Steinhardt, et al. 2006. "Variable Faint Optical Sources Discovered by Comparing the POSS and SDSS Catalogs." The Astronomical Journal 131 (6) (June): 2801-2825. doi:10.1086/503672.

\section{Published Version}

doi:10.1086/503672

\section{Permanent link}

http://nrs.harvard.edu/urn-3:HUL.InstRepos:33370048

\section{Terms of Use}

This article was downloaded from Harvard University's DASH repository, and is made available under the terms and conditions applicable to Other Posted Material, as set forth at http:// nrs.harvard.edu/urn-3:HUL.InstRepos:dash.current.terms-of-use\#LAA

\section{Share Your Story}

The Harvard community has made this article openly available.

Please share how this access benefits you. Submit a story.

\section{Accessibility}




\title{
VARIABLE FAINT OPTICAL SOURCES DISCOVERED BY COMPARING THE POSS AND SDSS CATALOGS
}

\author{
Branimir Sesar, ${ }^{1}$ Domjan Svilković, ${ }^{2}$ Željko Ivezić, ${ }^{1,3,4}$ Robert H. Lupton, ${ }^{3}$ Jeffrey A. Munn, ${ }^{5}$ Douglas Finkbeiner, ${ }^{3,4,6}$ \\ William Steinhardt, ${ }^{3}$ Rob Siverd, ${ }^{3}$ David E. Johnston, ${ }^{3}$ Gillian R. Knapp, ${ }^{3}$ James E. Gunn, ${ }^{3}$ Constance M. Rockosi, ${ }^{1}$ \\ David Schlegel, ${ }^{3}$ Daniel E. Vanden Berk, ${ }^{7}$ Pat Hall, ${ }^{3,8}$ Donald P. Schneider, ${ }^{9}$ and Robert J. Brunner ${ }^{10}$ \\ Received 2004 March 12; accepted 2006 February 17
}

\begin{abstract}
We present a study of variable faint optical sources discovered by comparing the Sloan Digital Sky Survey (SDSS) and the Palomar Observatory Sky Survey (POSS) catalogs. We use SDSS measurements to photometrically recalibrate several publicly available POSS catalogs (USNO-A2.0, USNO-B1.0, DPOSS [the Digitized Second Palomar Observatory Sky Survey], and GSC2.2 [the Guide Star Catalog 2.2]). A piecewise recalibration of the POSS data in $100 \operatorname{arcmin}^{2}$ patches (one SDSS field) generally results in an improvement of photometric accuracy (rms) by nearly a factor of 2, compared to the original data. In addition to the smaller core width of the error distribution, the tails of the distribution become much steeper after the recalibration. These improvements are mostly due to the very dense grid of calibration stars provided by SDSS, which rectifies the intrinsic inhomogeneities of Schmidt plates. We find that the POSS I magnitudes can be improved to $\sim 0.15$ mag accuracy, and POSS II magnitudes to $\sim 0.10$ mag accuracy. The smallest final errors are obtained with the GSC2.2 catalog, for which they approach 0.07 mag at the bright end. We use the recalibrated catalogs for the $\sim 3300 \mathrm{deg}^{2}$ of sky in the SDSS Data Release 2 to construct a catalog of $\sim 60,000$ sources that are variable on timescales of $10-50 \mathrm{yr}$, and make it publicly available. Using this catalog, we find that at least $1 \%$ of faint optical sources appear variable at the $>0.25$ mag level, and that about $20 \%$ of the variable population are quasars, although they represent only $0.6 \%$ of all point sources in the adopted flux-limited sample $(g<19)$. A series of statistical tests based on the morphology of SDSS color-magnitude and color-color diagrams, as well as visual comparison of images and comparison with repeated SDSS observations, demonstrate the robustness of the selection methods: three out of four candidate variable sources are correctly recognized to vary. We also demonstrate that candidate RR Lyrae stars trace the same halo structures, such as the Sgr dwarf tidal stream, that were discovered using repeated SDSS observations. We utilize the POSS-SDSS selected candidates to constrain the halo structure in the parts of sky for which repeated SDSS observations do not exist. We quantify the distribution of variable sources in the SDSS color-color diagrams and the variability characteristics of quasars. The observed long-term quasar variability (structure function) is smaller than predicted by the extrapolation of the power law measured for short timescales using repeated SDSS imaging ( 0.35 vs. 0.60 mag for SDSS-POSS I and 0.24 vs. $0.35 \mathrm{mag}$ for SDSS-POSS II, rms). This turnover in structure function suggests that the characteristic timescale for quasar variability is on the order of $1 \mathrm{yr}$ in the rest frame.
\end{abstract}

Key words: Galaxy: halo - Galaxy: stellar content — quasars: general — stars: variables: other

Online material: color figures

\section{INTRODUCTION}

The time domain represents another dimension, in addition to the spectral and spatial ones, in the exploration of celestial objects. Despite the importance of variability phenomena, the properties of optically faint variable sources are by and large unknown. There are about $10^{9}$ stars brighter than $V=20$ in the sky, and at least $3 \%$ of them are expected to be variable at the level of a

\footnotetext{
${ }^{1}$ Department of Astronomy, University of Washington, Box 351580, Seattle, WA 98195 .

${ }^{2}$ Department of Physics, University of Zagreb, Bijenička cesta 32, 10000 Zagreb, Croatia.

3 Princeton University Observatory, Princeton, NJ 08544.

${ }^{4}$ H. N. Russell Fellow.

${ }^{5}$ US Naval Observatory, Flagstaff Station, P.O. Box 1149, Flagstaff, AZ 86002

${ }_{7}^{6}$ Hubble Fellow.

${ }^{7}$ Department of Physics and Astronomy, University of Pittsburgh, 3941 O'Hara Street, Pittsburgh, PA 15260.

${ }^{8}$ Departamento de Astronomía y Astrofísica, Pontificia Universidad Católica de Chile, Facultad de Física, Casilla 306, Santiago 22, Chile.

${ }_{9}$ Department of Astronomy and Astrophysics, Pennsylvania State University, University Park, PA 16802.

${ }_{10}$ National Center for Supercomputing Applications and Department of Astronomy, University of Illinois, Urbana, IL 61801.
}

few percent (Eyer 1999). However, the overwhelming majority are not recognized as variable sources even at the brightest magnitudes: $90 \%$ of variable stars with $V<12$ remain to be discovered (Paczyński 2000). Paczyński (1997) lists striking examples of the serious incompleteness in the available samples of variable stars: eclipsing binaries of the Algol type and contact binaries (W UMa stars) are incomplete fainter than $V \sim 12$, and RS CVn type binaries are complete only to $V \sim 5$. Another vivid example of serious selection effects is the sky distribution of RR Lyrae stars: objects listed in the fourth General Catalog of Variable Stars (the main resource for variable stars) are distributed in isolated square patches with the size and shape of the Schmidt plates used to discover them.

The discrepancy between the utility of variable stars and the available observational data has prompted several contemporary projects aimed at regular monitoring of the optical sky. The current state of the art has also greatly benefited from past and present microlensing searches (Paczyński 2001). We list here some of the more prominent surveys in terms of sky coverage, depth, and cadence.

1. The Faint Sky Variability Survey (Groot et al. 2003) is a very deep $(V=17-24) B V I$ survey of $23 \mathrm{deg}^{2}$ of sky, containing about 80,000 sources. 


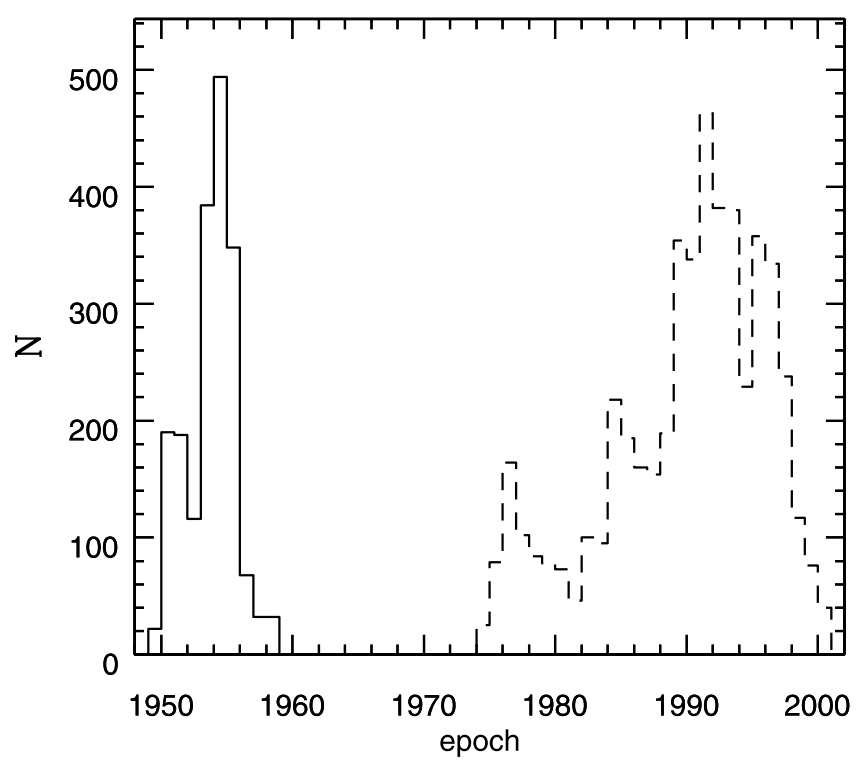

FIG. 1.-Distribution of epochs for the POSS I (solid line) and POSS II (dashed line) surveys. [See the electronic edition of the Journal for a color version of this figure.]

2. The QUEST (Quasar Equatorial Survey Team) survey (Vivas et al. 2001) monitors $700 \operatorname{deg}^{2}$ of sky from $V=13.5$ to a limit of $V=21$.

3. ROTSE (Robotic Optical Transient Search Experiment; Akerlof et al. 2000) monitors the entire observable sky twice a night from $V=10$ to a limit of $V=15.5$.

4. OGLE (Optical Gravitational Lensing Experiment; most recently OGLE III [Udalski et al. 2002]) monitors $\sim 100 \mathrm{deg}^{2}$ toward the Galactic bulge from $I=11.5$ to a limit of $I=20$. Due to a very high stellar density toward the bulge, OGLE II has detected over 200,000 variable stars (Woźniak et al. 2002).

These and other surveys have demonstrated that in addition to variable stars, there are many other exciting photometrically variable objects in the sky. For example, ROTSE I detected an optical flash generated by a gamma-ray burst at a redshift of 1.6, the most luminous optical source ever measured $\left(V=9, M_{V}=\right.$ -36.4 ; Vestrand et al. 2002). The detection of such optical flashes may place strong constraints on the physical mechanisms responsible for gamma-ray bursts. Similarly, the variability of quasars offers significant clues for the origin of their emission (e.g., Trevese et al. 2001).

Recognizing the outstanding importance of variable objects, the last Decadal Survey Report highly recommended a major new initiative for studying the variable sky, the Large Synoptic Survey Telescope (LSST). The LSST ${ }^{11}$ will offer an unprecedented view of the faint-variable sky: according to the current designs it will scan the entire accessible sky every three nights to a limit of $V \sim 24$. Compared to any other survey currently available, the data from LSST will be revolutionary. Yet, at least half a decade or more will elapse before the first photons are detected by the LSST. Meanwhile, the already available Palomar Observatory Sky Surveys (POSS I and POSS II; for references see Appendix A) and the ongoing Sloan Digital Sky Survey (SDSS; see $\S 2.2$ ) can be used to study the properties of faint $(r \sim 20)$ optical sources, and here we present such a study.

\footnotetext{
${ }^{11}$ There are currently two designs considered for implementation: a distributedaperture approach (Pan-STARRS; see http://pan-starrs.ifa.hawaii.edu; Kaiser et al. 2002) and a single large-aperture telescope (see http://www.lsst.org; Tyson 2002).
}

TABLE 1

Best-Fit Coefficients for POSS-SDSS

Photometric Transformations

\begin{tabular}{|c|c|c|c|}
\hline Band & $b$ & $c$ & $\sigma$ \\
\hline$O_{a} \ldots \ldots \ldots \ldots$ & 0.354 & -0.32 & 0.26 \\
\hline$E_{a} \ldots \ldots \ldots \ldots$ & -0.101 & -0.30 & 0.25 \\
\hline$O \ldots \ldots \ldots \ldots$ & 0.444 & 0.05 & 0.31 \\
\hline$E \ldots \ldots \ldots \ldots \ldots$ & -0.162 & -0.29 & 0.27 \\
\hline$J$ & 0.075 & 0.10 & 0.32 \\
\hline$F$ & -0.133 & -0.14 & 0.20 \\
\hline$N \ldots \ldots \ldots \ldots \ldots$ & -0.530 & -0.37 & 0.25 \\
\hline$J_{g} \ldots \ldots \ldots \ldots$ & 0.105 & 0.20 & 0.14 \\
\hline$F_{g} \ldots \ldots \ldots \ldots \ldots$ & -0.101 & -0.18 & 0.10 \\
\hline$G \ldots \ldots \ldots \ldots \ldots$ & -0.392 & -0.28 & 0.20 \\
\hline$R$ & -0.127 & 0.10 & 0.17 \\
\hline$I \ldots \ldots \ldots$ & -0.270 & 0.32 & 0.27 \\
\hline
\end{tabular}

Note.-For the definitions of $b$ and $c$, see eq. (1).

The fourth column lists the residual rms scatter.

The comparison of the POSS and SDSS surveys allows studies of long-term variability with timescales of up to half a century. By necessity, such studies are based on a small number of observations of the same objects to constrain the ensemble properties of a sample of sources, as opposed to studying well-sampled light curves for a small number of objects. The lack of detailed information for individual objects is compensated in some ways by the large sample size. In addition, the five-band-accurate SDSS photometry can be used for very detailed source classification, e.g., separation of quasars and stars (Richards et al. 2002), spectral classification of stars to within one or two spectral subtypes (Lenz et al. 1998; Finlator et al. 2000; Hawley et al. 2002), and even remarkably efficient color selection (thanks to accurate $u$-band photometry) of the low-metallicity $\mathrm{G}$ and $\mathrm{K}$ giants (Helmi et al. 2003) and horizontal-branch stars (Yanny et al. 2000; Ivezić et al. 2005).

However, when using only several observations, the robustness of variability detection critically depends on the stability of the photometric errors. While the SDSS photometric errors are small ( 0.02) and well behaved (Ivezić et al. 2003, 2004a), older photographic POSS data can have large errors (tenths of magnitudes) due to intrinsic inhomogeneities of Schmidt plates and the lack of a sufficient number of calibration stars. This problem can be alleviated to some extent by using photometric measurements of stars in the SDSS to recalibrate the POSS catalogs. In $\S 2$ we describe such a recalibration method and demonstrate that photometric errors in the POSS catalogs can be decreased by a factor of $\sim 2$ (rms), with a significant improvement in the behavior of the error distribution tails. In $\S 3$ we use SDSS data and recalibrated POSS catalogs to select variable objects in $\sim 3300 \mathrm{deg}^{2}$ of sky from the SDSS Data Release 2 (Abazajian et al. 2004). In the same section we discuss tests that demonstrate the robustness of the selection algorithm, and quantify the distribution of variable sources in the SDSS color-color diagrams. The Milky Way halo structure traced by selected candidate RR Lyrae stars is discussed in $\S 4$, and in $\S 5$ we analyze the variability of quasars. Our main results are summarized in $\S 6$.

\section{THE PHOTOMETRIC RECALIBRATION OF POSS CATALOGS USING SDSS MEASUREMENTS}

\subsection{The Input POSS Catalogs}

We utilize several publicly available POSS catalogs: USNOA2.0, USNO-B1.0, GSC2.2 (the Guide Star Catalog 2.2), and DPOSS (the Digitized Second Palomar Observatory Sky Survey). 


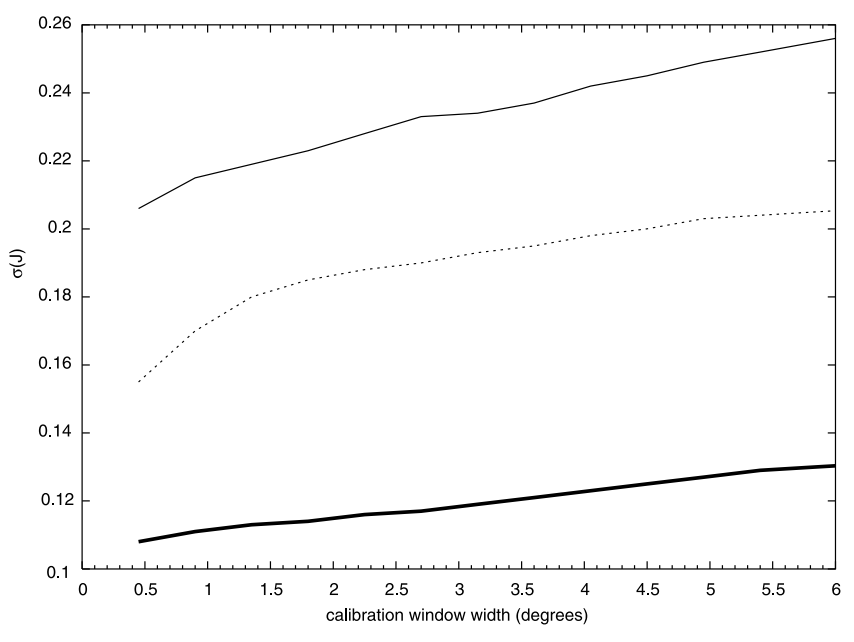

FIG. 2.-Photometric accuracy as a function of the recalibration window width for three randomly chosen POSS II $J$ plates. The decrease of the window width decreases photometric errors, as expected. Note that when extrapolating curves to zero window width, plates show varying photometric accuracy, reflecting different intrinsic properties. The estimated improvement in accuracy by decreasing the window width from $\sim 0.5$ to $0^{\circ}$ is only $\sim 0.01-0.02 \mathrm{mag}$. [See the electronic edition of the Journal for a color version of this figure.]

A description of each catalog and references are listed in Appendix A. Here we briefly mention that all four catalogs utilize the same POSS I and POSS II Schmidt plates. However, the scanning and calibration procedures are different, and the source parameters, such as magnitudes, reported in different catalogs in gen- eral are not the same for the same sources detected on the same plates (see $\S 2.5 .3$ for more details). USNO-A2.0 reports $O$ and $E$ magnitudes, hereafter $O_{a}$ and $E_{a}$ to distinguish them from the $O$ and $E$ magnitudes reported in the USNO-B1.0 catalog. The latter catalog also lists $J, F$, and $N$ magnitudes. The GSC2.2 catalog lists $J$ and $F$ magnitudes, hereafter $J_{g}$ and $F_{g}$ to distinguish them from the $J$ and $F$ magnitudes reported in the USNO-B1.0 catalog. The DPOSS catalog is also based on photographic $J$, $F$, and $N$, but they are calibrated and reported as $G, R$, and I magnitudes.

The completeness of the USNO-B1.0 catalog, measured using SDSS data, is discussed by Munn et al. (2004). Our analysis of other catalogs confirms their result that, in general, POSS catalogs are $\sim 95 \%$ complete at magnitudes brighter than 19-20 (depending on a particular band/catalog), and have faint limits (which we define as the magnitude at which fewer than $50 \%$ of SDSS sources are found in a POSS catalog) at 20.5-21. The distribution of epochs for POSS I and POSS II is shown in Figure 1.

\subsection{Sloan Digital Sky Survey}

The SDSS is a digital photometric and spectroscopic survey that will cover up to one-quarter of the celestial sphere in the north Galactic cap and produce a smaller area $\left(\sim 225 \mathrm{deg}^{2}\right)$ but much deeper survey in the southern Galactic hemisphere ${ }^{12}$ (York et al. 2000; Stoughton et al. 2002; Abazajian et al. 2004). The flux densities of detected objects are measured almost simultaneously

\footnotetext{
12 See also http://www.astro.princeton.edu/PBOOK/welcome.htm.
}
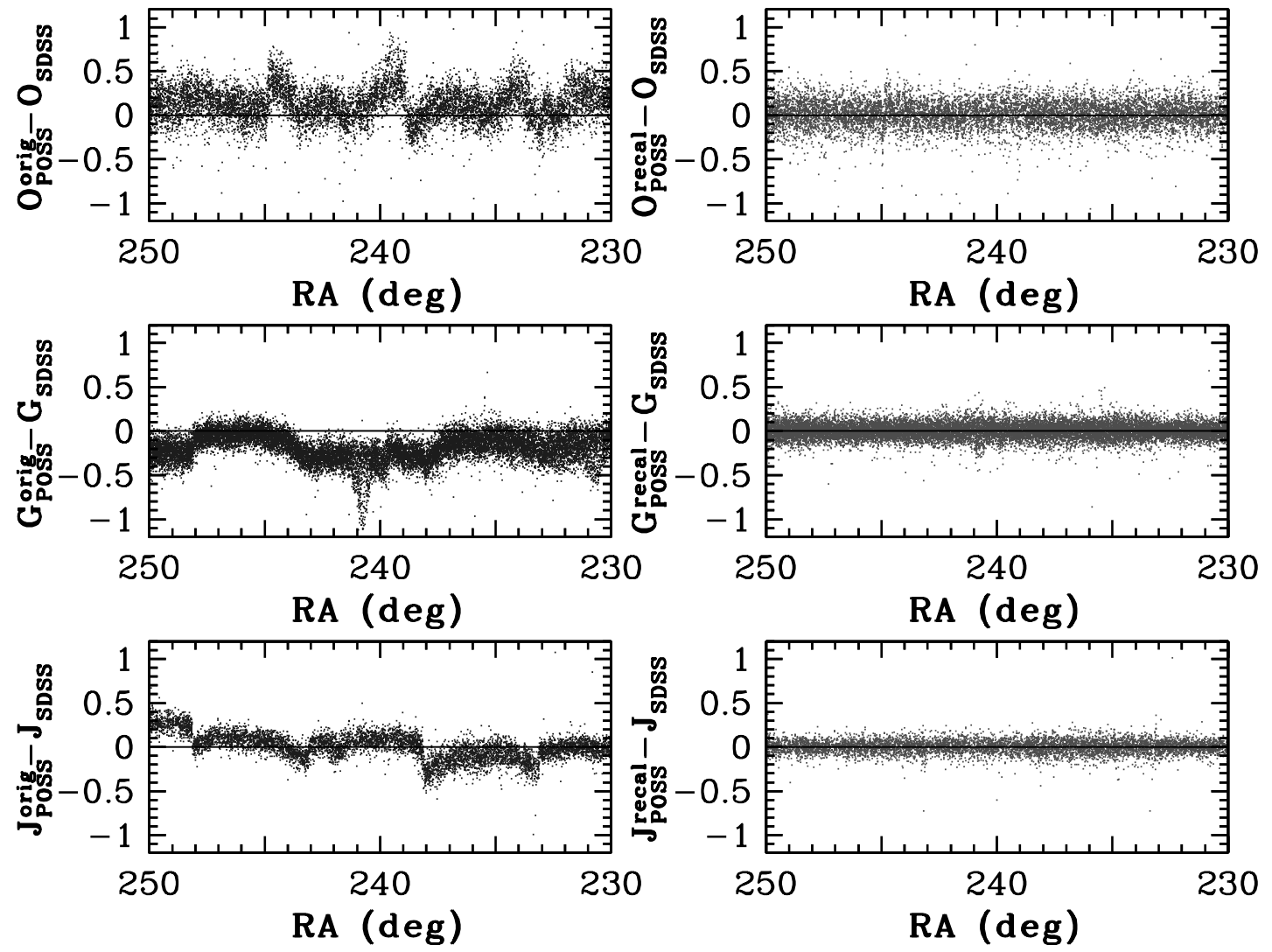

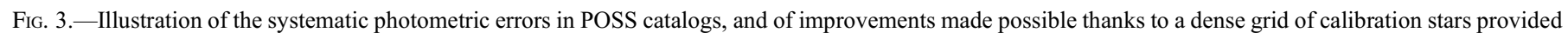

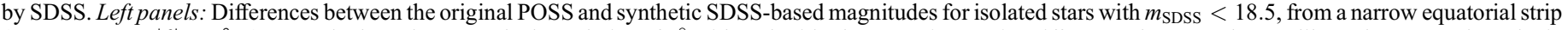

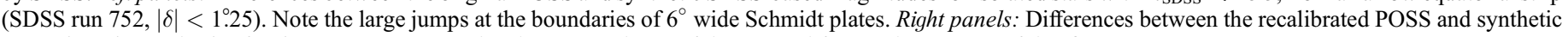
SDSS-based magnitudes for the same stars. [See the electronic edition of the Journal for a color version of this figure.] 


\section{USNO-A2.0 vs. SDSS run 752}
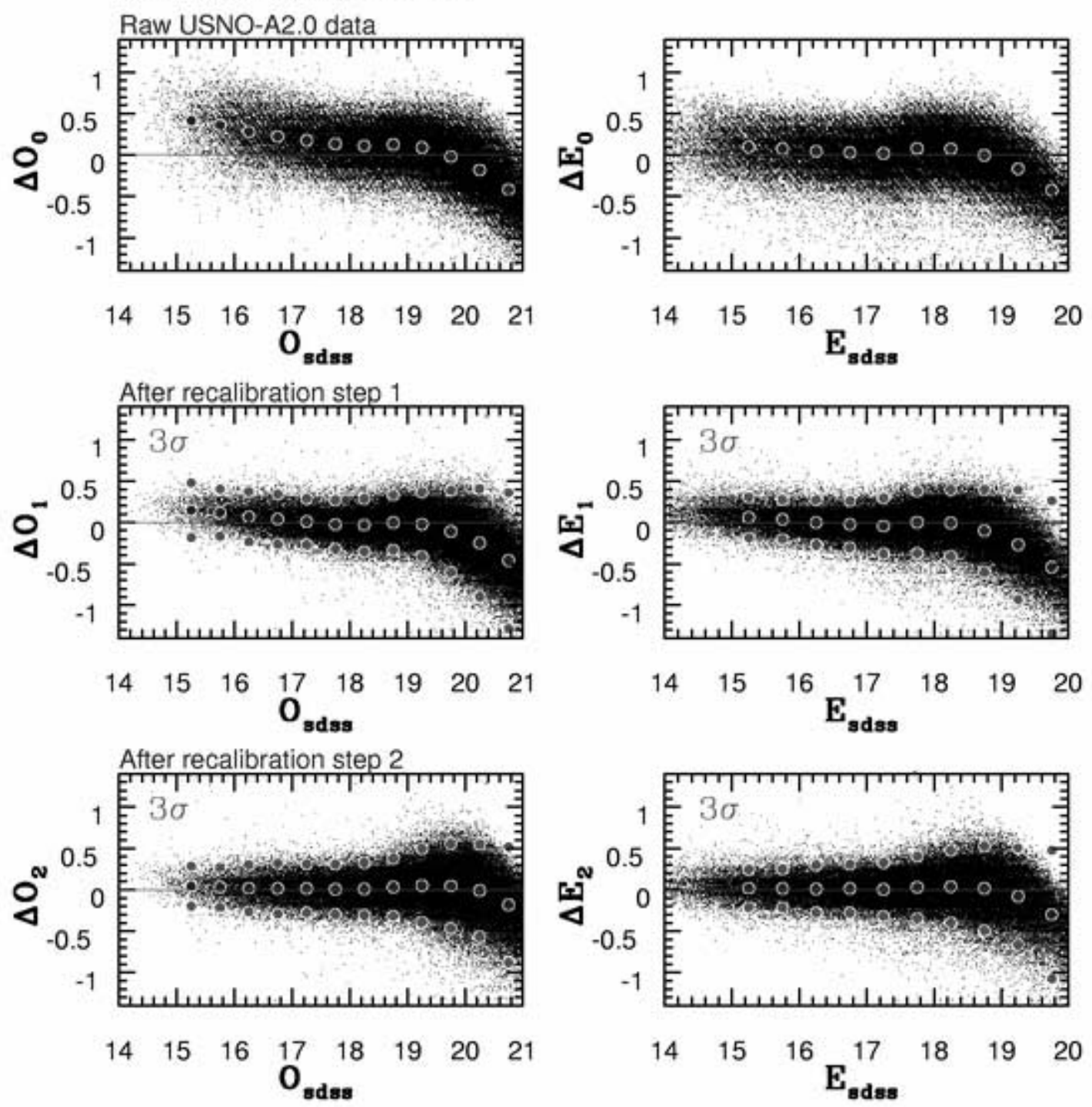

FIG. 4.- Illustration of the recalibration method for the USNO-A2.0 catalog. The dots in the top panels represent magnitude differences between the original POSS $O$ (left) and $E$ (right) magnitudes and the synthetic SDSS-based $O$ and $E$ magnitudes, as a function of the latter, for about 300,000 stars observed in $\sim 100$ deg ${ }^{2}$ of sky in SDSS run 752. The middle panels show the magnitude differences after the first recalibration step, where color-term and zero-point systematic errors are removed. The results of the second recalibration step, which removes the dependence of magnitude differences on magnitude, are shown in the bottom panels. The middle set of large symbols in each panel shows the median differences in magnitude bins, and the two outer sets of large symbols show the equivalent Gaussian widths (determined from the interquartile range), multiplied by 3. [See the electronic edition of the Journal for a color version of this figure.]

in five bands $(u, g, r, i$, and $z)$ with effective wavelengths of 3540 , 4760, 6280, 7690, and $9250 \AA$ (Fukugita et al. 1996; Gunn et al. 1998; Smith et al. 2002; Hogg et al. 2001). The completeness of the SDSS catalogs for point sources is $\sim 99.3 \%$ at the bright end (Ivezić et al. 2001) and drops to $95 \%$ at limiting magnitudes ${ }^{13}$ of $22.1,22.4,22.1,21.2$, and 20.3 (the SDSS saturation limit is $\sim 14$ in the $r$ band and somewhat brighter in other bands). All magnitudes are given in the $\mathrm{AB}_{\nu}$ system (Oke \& Gunn 1983; for additional discussion regarding the SDSS photometric system, see Fukugita et al. [1996] and Fan [1999]). The survey sky coverage of about $\pi \mathrm{sr}\left(10,000 \mathrm{deg}^{2}\right)$ will result in photometric measurements to the above detection limits for about 100 million stars and a similar number of galaxies. Astrometric positions are accurate to

${ }^{13}$ These values are determined by comparing multiple scans of the same area obtained during the commissioning year. Typical seeing in these observations was $1 . " 5 \pm 0.1$. about 0 .' 1 per coordinate for sources brighter than $r \sim 20.5$ (Pier et al. 2003), and the morphological information from the images allows robust star-galaxy separation to $r \sim 21.5$ (Lupton et al. 2002). More technical details can be found in Stoughton et al. (2002) and on the SDSS World Wide Web site. ${ }^{14}$

In this work we use the SDSS Data Release 2, which provides data for $3324 \mathrm{deg}^{2}$ of the sky. The equatorial Aitoff projection of this area can be found at the SDSS Web site (see also Fig. 1 of Ivezić et al. 2004c).

\subsection{Photometric Transformations between POSS and SDSS Systems}

We chose to synthesize magnitudes in the POSS bands using SDSS measurements and then recalibrate the POSS catalogs using their original bands. The alternative of recalibrating POSS

\footnotetext{
${ }^{14}$ See http://www.sdss.org
} 

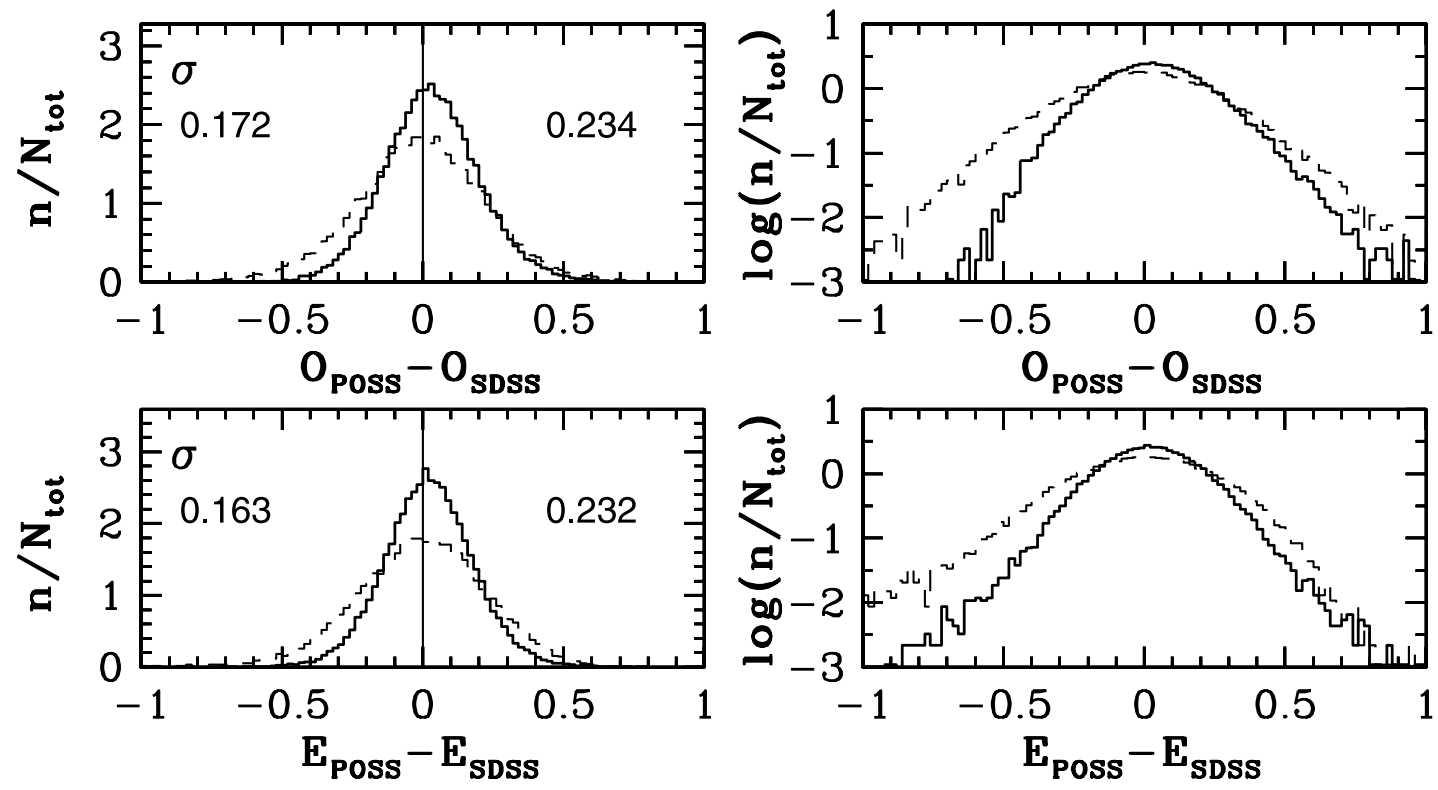

FIG. 5.-Improvements in photometric errors after recalibration for the USNO-A2.0 catalog. The POSS-SDSS magnitude differences before recalibration are shown by dashed lines, and those after recalibration by solid lines. The left panels show error distributions on a linear scale, and the right panels on a logarithmic scale. The equivalent Gaussian widths, determined from the interquartile range, are shown in the left panels (right value, before; left value, after). [See the electronic edition of the Journal for a color version of this figure.]

catalogs directly to the SDSS system is less desirable because colors at the POSS epoch are poorly known, and this may have an effect on the photometric accuracy for variable sources. Following Monet et al. (2003), we adopt the following form to define synthetic POSS magnitudes, $m_{\mathrm{SDSS}}$, calculated from SDSS photometry:

$$
m_{\mathrm{SDSS}}=m+b \text { color }+c
$$

where $m=g, r, g, r, i, g, r$, and $i$ and color $=g-r, g-r, g-r$, $g-r, r-i, g-r, g-r$, and $r-i$ for $O, E, J, F, N, G, R$, and $I$, respectively [e.g., $\left.O_{\text {SDSS }}=g+b(g-r)+c\right]$. Utilizing data for about $\sim 300 \mathrm{deg}^{2}$ of sky (SDSS runs 752 and 756), we derived the best-fit values of coefficients $b$ and $c$ for each band and the POSS catalog. We used only "good" sources, defined as follows:

1. Sources must be unresolved in SDSS data (note that the SDSS star-galaxy separation is robust to at least $r \sim 21.5$, which is significantly fainter than the faint limit of the resulting sample).

2. Sources must be isolated in SDSS data. This condition ensures that the USNO/GSC/DPOSS photometry is not affected by difficult-to-measure, blended objects.

3. Sources must not be saturated in the $g$ or $r$ band in the SDSS data (roughly equivalent to $g, r>14$ ) and must have $g<19$ to ensure good photon statistics in the POSS sample.

4. The USNO/GSC/DPOSS and SDSS positions must agree to better than $2^{\prime \prime}$. This limit corresponds to a $\sim 5 \sigma$ cut on astrometric errors (Pier et al. 2003).

5. The sources must have $u-g>0.7$ (measured by SDSS) to avoid highly variable quasars (see $\S 5$ ).

The best-fit values of coefficients $b$ and $c$ and the residual rms scatter (which is a good measure of the mean photometric accuracy of the POSS catalogs) are listed in Table 1. Note that $J$ and $F$ magnitudes from the GSC2.2 catalog have the smallest residual scatter, while the $O$ and $E$ magnitudes have the largest scatter.

Similar values for the $b$ and $c$ coefficients were derived for the USNO-B1.0 magnitudes by Monet et al. (2003). We have verified that adopting their transformations results in only slightly larger residual scatter (by $\sim 0.01-0.02 \mathrm{mag}$ ) for the other three catalogs. Thus, in order to prevent proliferation of various SDSS-POSS transformations, we adopt their transformations, which we list here for completeness, in the rest of this work:

$$
\begin{aligned}
& O_{\mathrm{SDSS}}=g+0.452(g-r)+0.08 \\
& E_{\mathrm{SDSS}}=r-0.086(g-r)-0.20 \\
& J_{\mathrm{SDSS}}=g+0.079(g-r)+0.06 \\
& F_{\mathrm{SDSS}}=r-0.109(g-r)-0.09 \\
& N_{\mathrm{SDSS}}=i-0.164(r-i)-0.44
\end{aligned}
$$

Since SDSS-POSS transformations for DPOSS $G, R$, and $I$ magnitudes $^{15}$ are not available, we adopt transformations similar to those used by Monet et al. (2003) and use the best-fit values of coefficients $b$ and $c$ from Table 1:

$$
\begin{aligned}
G_{\mathrm{SDSS}} & =g-0.392(g-r)-0.28 \\
R_{\mathrm{SDSS}} & =r-0.127(g-r)+0.10 \\
I_{\mathrm{SDSS}} & =i-0.270(r-i)+0.32
\end{aligned}
$$

\subsection{The Recalibration Method}

The first basic premise of the recalibration method employed here is that the SDSS photometric errors are negligible compared to errors in the POSS catalogs: the SDSS photometric errors are $\sim 0.02 \mathrm{mag}$, as demonstrated by repeated scans (Ivezić et al. 2003), while the errors in the POSS catalogs are 0.1 mag or larger. The second premise is that not more than a few percent of faint stars vary by more than a few percent, in agreement with the available data and models (Eyer 1999). The third assumption is that systematic errors are a significant contribution to photometric errors in the POSS catalog and thus can be calibrated out using a

\footnotetext{
${ }^{15}$ We use uppercase letters for DPOSS magnitudes to distinguish them from SDSS $g, r$, and $i$ magnitudes.
} 

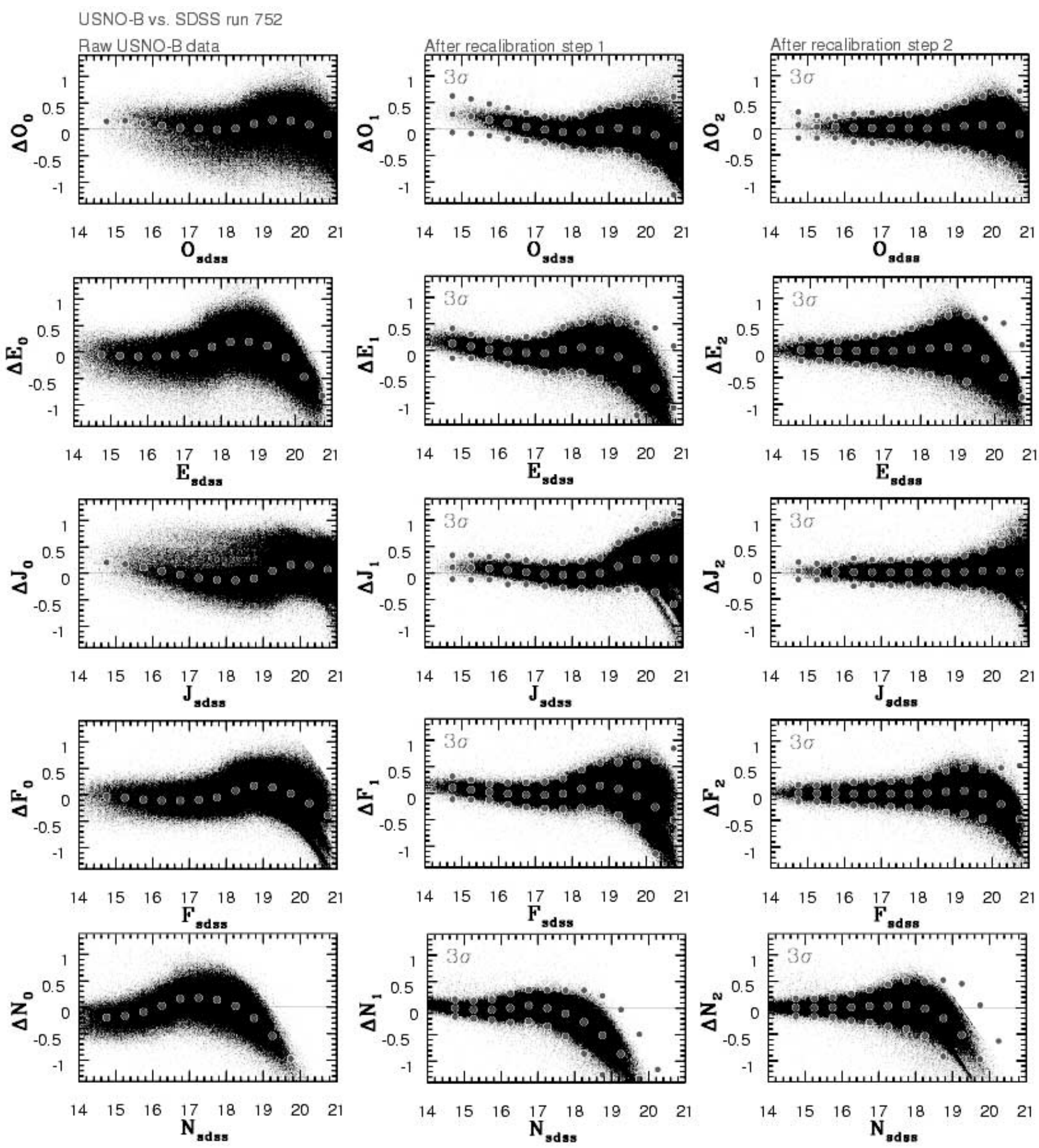

FIG. 6.-Same as Fig. 4, except for the USNO-B1.0 catalog. [See the electronic edition of the Journal for a color version of this figure.]

dense grid of calibration stars provided by SDSS. We demonstrate empirically that indeed the accuracy of POSS-based photometry can be improved by about a factor of 2 in all analyzed catalogs.

The recalibration of the POSS catalogs is performed in two steps. In the first step the subsamples of good objects (see $\S 2.3$ ) are grouped by Schmidt plate and SDSS field. One SDSS field has an area of $0.034 \mathrm{deg}^{2}$; this is sufficiently large to include enough calibration stars (typically 50-200), and yet sufficiently small that the response of the Schmidt plates is nearly constant, as shown by Lattanzi \& Bucciarelli (1991). To avoid edge effects, we use a running window with the width of three SDSS fields ( 0.45 ; see $\S 2.4 .1$ for more details).

For each of the five POSS magnitudes, we minimize $\sum\left(m_{\text {recalib }}-m_{\mathrm{SDSS}}\right)^{2}$ using the least-squares method, where

$$
m_{\text {recalib }}=A m_{\mathrm{POSS}}+B \text { color }+C
$$

and $m=O, E, J, F, N, G, R$, and $I\left(m_{\mathrm{SDSS}}\right.$ is defined by eqs. [2] and [3]). This step removes systematic magnitude errors due to local nonlinearities of the plate, color-term dependence, and zero-point 

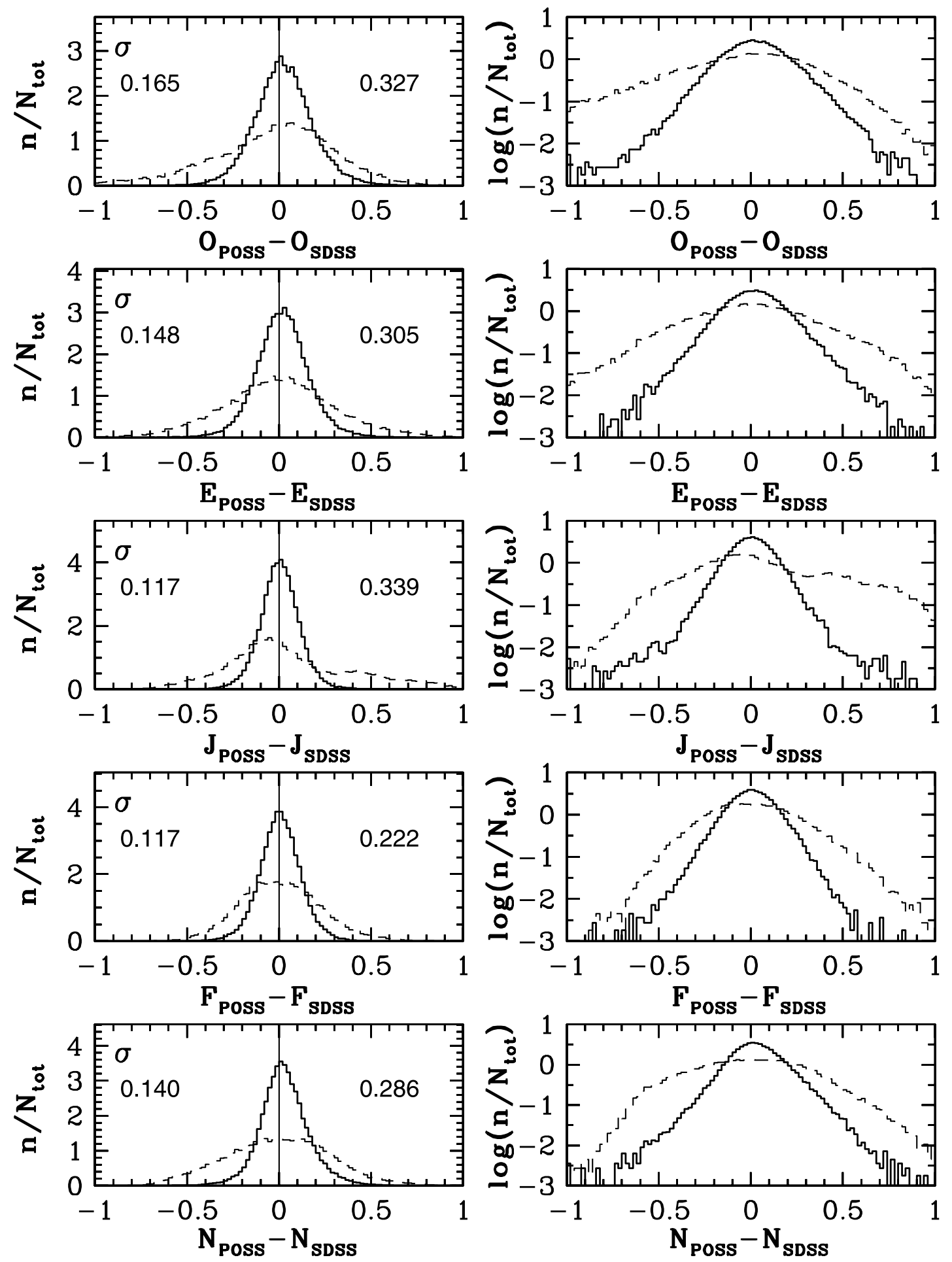

FIG. 7.- Same as Fig. 5, except for the USNO-B1.0 catalog. [See the electronic edition of the Journal for a color version of this figure.]

offsets. ${ }^{16}$ In the second step, we use all the good sources from a given Schmidt plate $\left(\sim 36 \mathrm{deg}^{2}\right)$ to correct the dependence of the $m_{\text {recalib }}-m_{\text {SDSS }}$ residuals on magnitude (using median $m_{\text {recalib }}-m_{\text {SDSS }}$ in 1 mag wide bins, and linear interpolation between the bin centers). Such residuals are typically larger at the faint end and are probably caused by incorrect sky estimates in the POSS catalogs. Also, for all objects on a given Schmidt

\footnotetext{
${ }^{16}$ A similar procedure was used by Munn et al. (2004) for astrometric recalibration of POSS catalogs.
}

plate, the photometric error $\sigma$ was determined using a fifth-degree polynomial fit to the standard deviation of $m_{\text {recalib }}-m_{\text {SDSS }}$ as a function of magnitude (in $0.5 \mathrm{mag}$ wide bins).

\subsubsection{The Optimal Recalibration Scale}

As advocated by Lattanzi \& Bucciarelli (1991), the characteristic scale for inhomogeneities in Schmidt plates is about 0.5 . We tested their result by recalibrating POSS II $J$-band plates by varying the calibration window width. Figure 2 shows the final errors as a function of that width for three randomly chosen plates. 

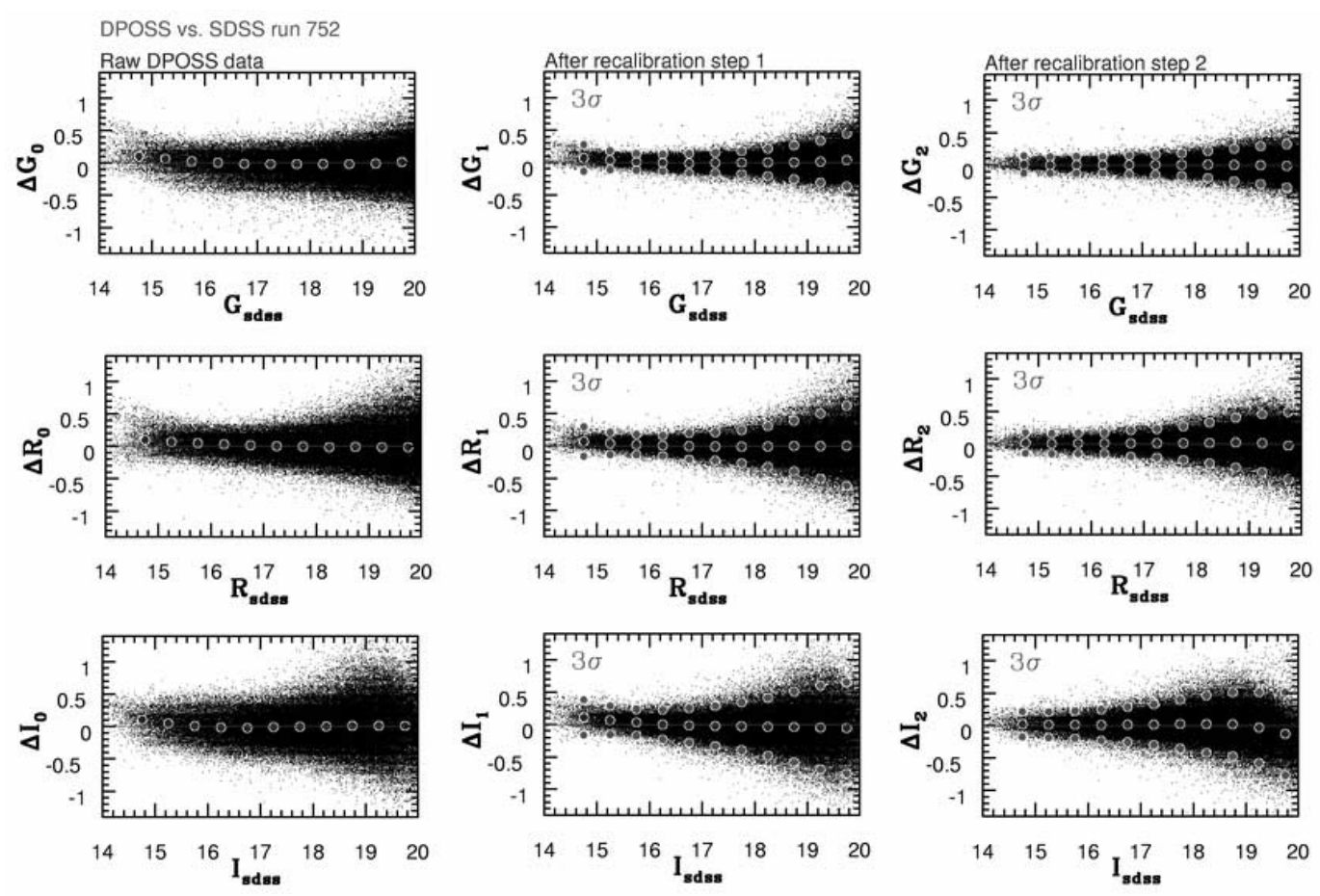

FIG. 8.-Same as Fig. 4, except for the DPOSS catalog. [See the electronic edition of the Journal for a color version of this figure.]
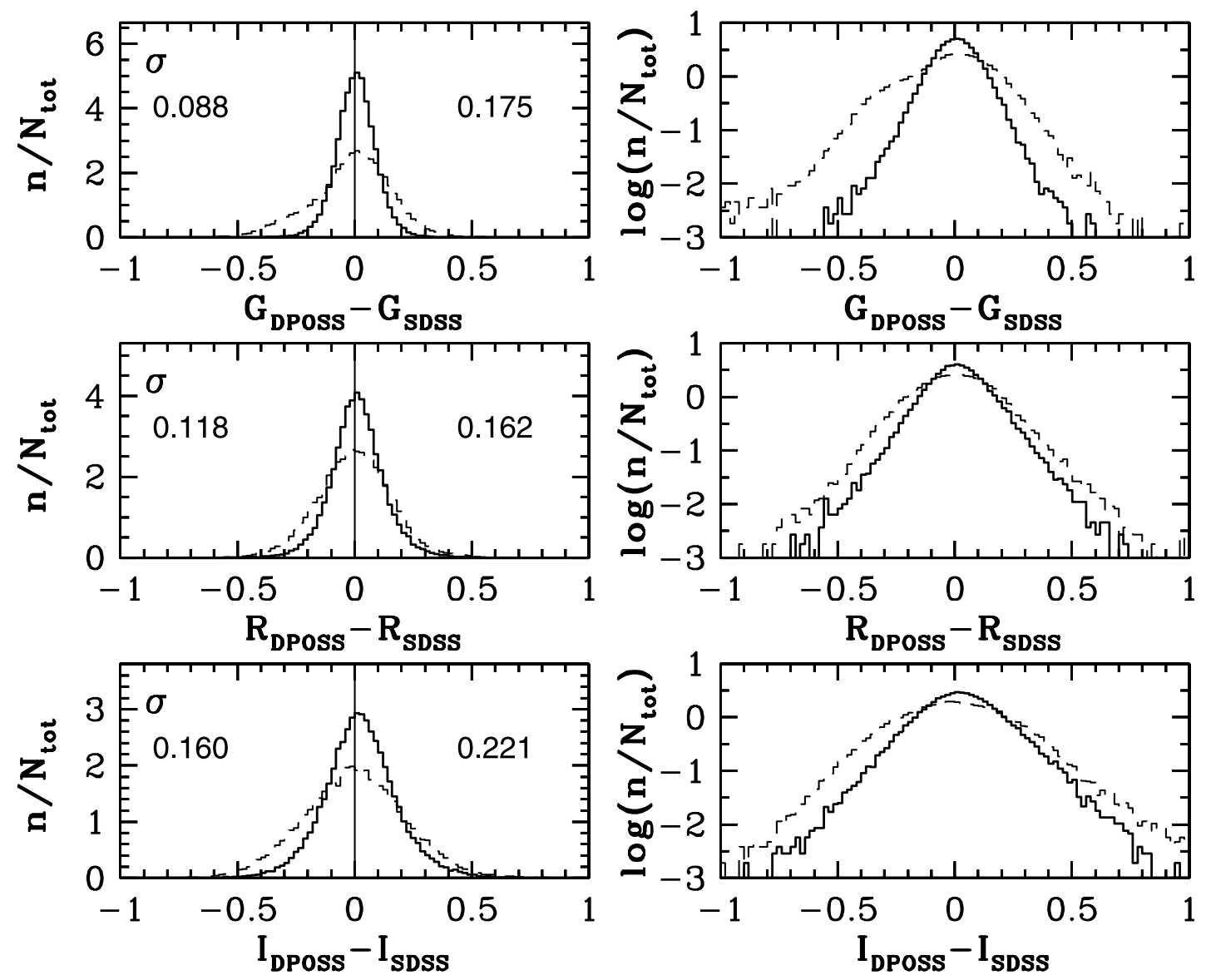

FIG. 9.-Same as Fig. 5, except for the DPOSS catalog. [See the electronic edition of the Journal for a color version of this figure.] 
TABLE 2

SumMary of IMPRovements IN POSS Photometry

\begin{tabular}{|c|c|c|c|c|c|c|c|c|c|c|}
\hline Catalog & $\sigma_{O}^{\text {old }}$ & $\sigma_{O}^{\text {new }}$ & $\sigma_{E}^{\text {old }}$ & $\sigma_{E}^{\text {new }}$ & $\sigma_{J}^{\text {old }}$ & $\sigma_{J}^{\text {new }}$ & $\sigma_{F}^{\text {old }}$ & $\sigma_{F}^{\text {new }}$ & $\sigma_{N}^{\text {old }}$ & $\sigma_{N}^{\text {new }}$ \\
\hline USNO-A2.0............ & 0.234 & 0.172 & 0.232 & 0.163 & . & & & .. & $\ldots$ & \\
\hline USNO-B1.0.............. & 0.327 & 0.165 & 0.305 & 0.148 & 0.339 & 0.117 & 0.222 & 0.117 & 0.286 & 0.140 \\
\hline GSC2.2 & $\ldots$ & $\ldots$ & $\ldots$ & $\ldots$ & 0.126 & 0.071 & 0.094 & 0.072 & $\ldots$ & .. \\
\hline DPOSS $^{\mathrm{a}} \ldots \ldots \ldots \ldots$ & $\ldots$ & $\ldots$ & $\ldots$ & $\ldots$ & 0.175 & 0.088 & 0.162 & 0.118 & 0.221 & 0.160 \\
\hline
\end{tabular}

Notes.- "Old" refers to rms scatter before recalibration, and "new" to rms scatter after recalibration.

a $J, F$, and $N$ bands listed for DPOSS correspond to $G, R$ and $I$ bands.

As expected, the decrease of calibration window width decreases photometric errors all the way to the practical limit of $\sim 0.5$ set by the minimum number of required calibration stars. The figure demonstrates that the improvement in accuracy by decreasing the window width from $\sim 0.5-1^{\circ}$ to $0^{\circ}$ is only $\sim 0.01 \mathrm{mag}$, thus confirming the result of Lattanzi \& Bucciarelli. Note that when extrapolating curves to zero window width, plates show varying photometric accuracy, reflecting different intrinsic properties.

The plate-dependent systematic photometric errors in the POSS catalogs are illustrated in Figure 3 (the behavior for the USNO-A2.0 catalog is similar). The large jumps in photometric errors at the boundaries of $6^{\circ}$ wide Schmidt plates are obvious and suggest that the photometric recalibration of POSS data is mandatory when searching for variable sources that vary less than a few tenths of a magnitude.

\subsection{Analysis of the Recalibration Results}

\subsubsection{Recalibration Results for the USNO Catalogs}

The results of the two recalibration steps, described in $\S 2.4$, are illustrated for the USNO-A2.0 catalog in Figure 4. As evident from the middle panels, the first step results in smaller scatter between SDSS and recalibrated POSS magnitudes, but the magnitude dependence of their differences remains appreciable. This dependence is removed in the second recalibration step, as discernible from the bottom panels.

The recalibration procedure generally results in about a factor of 2 improvement in the rms scatter between SDSS-based synthetic POSS magnitudes and the measured POSS magnitudes. Figure 5 compares the POSS-SDSS magnitude differences before (dashed lines) and after (solid lines) calibration, for the $O$ and $E$ bands, on a linear and logarithmic scale. As evident, the recalibration not only results in a smaller rms scatter, but also significantly clips the tails. Both effects are of crucial importance when selecting variable objects.

The corresponding results for the USNO-B1.0 catalog are shown in Figures 6 and 7. We note that the original $O$ and $E$ magnitudes have somewhat smaller errors in the USNO-A2.0 catalog, but the USNO-B1.0 magnitudes are slightly better after recalibration.

\subsubsection{Recalibration Results for the DPOSS and the GSC2.2 Catalogs}

The DPOSS catalog was recalibrated in the same manner as the USNO catalogs. When compared with USNO-B $J$ and $F$ bands, the DPOSS $G$ and $R$ bands do not show as strong a

\section{GSC2.2 vs. SDSS run 752}
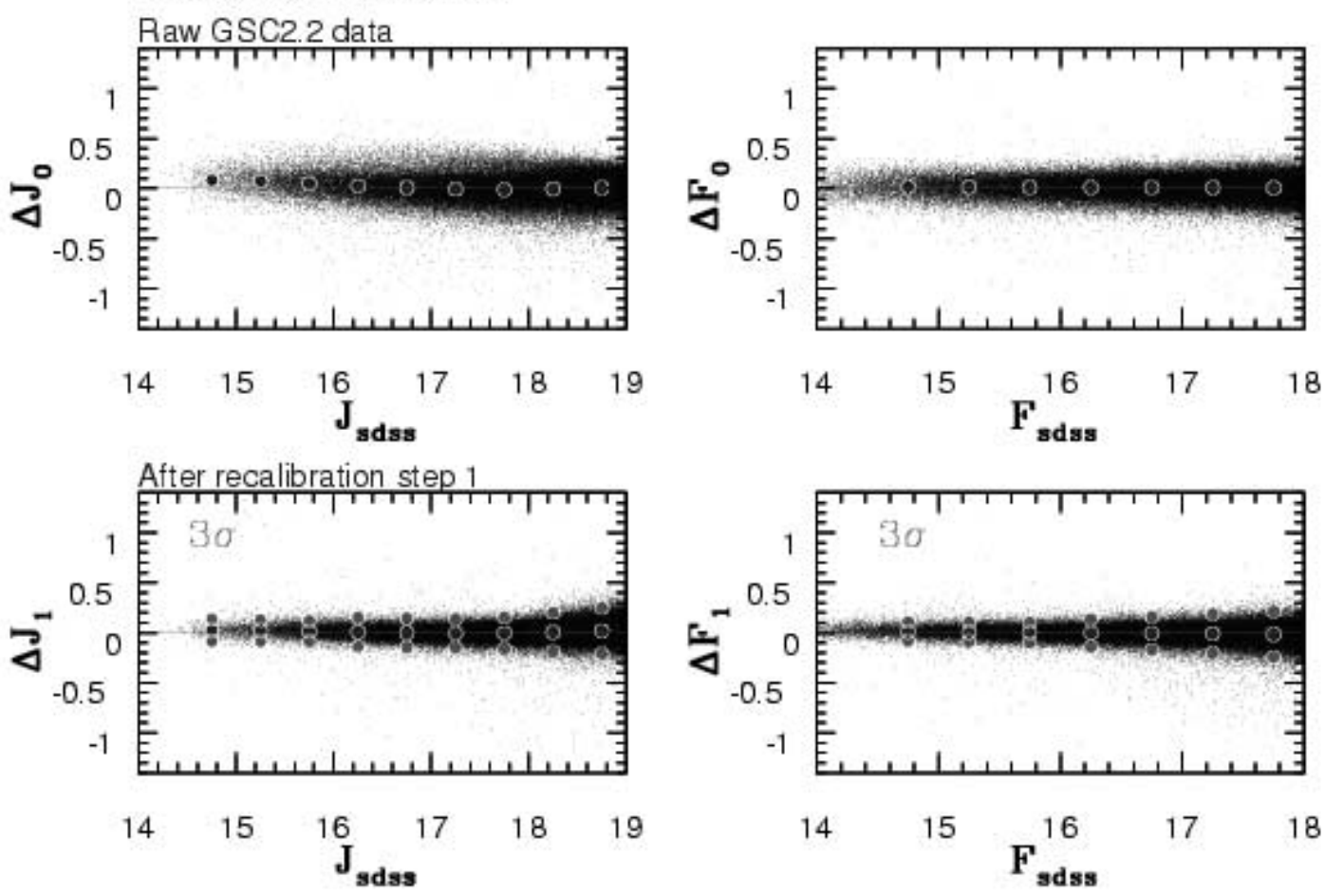

FIG. 10.-Same as Fig. 4, except that only the first recalibration step is shown, for the GSC2.2 catalog. [See the electronic edition of the Journal for a color version of this figure.] 

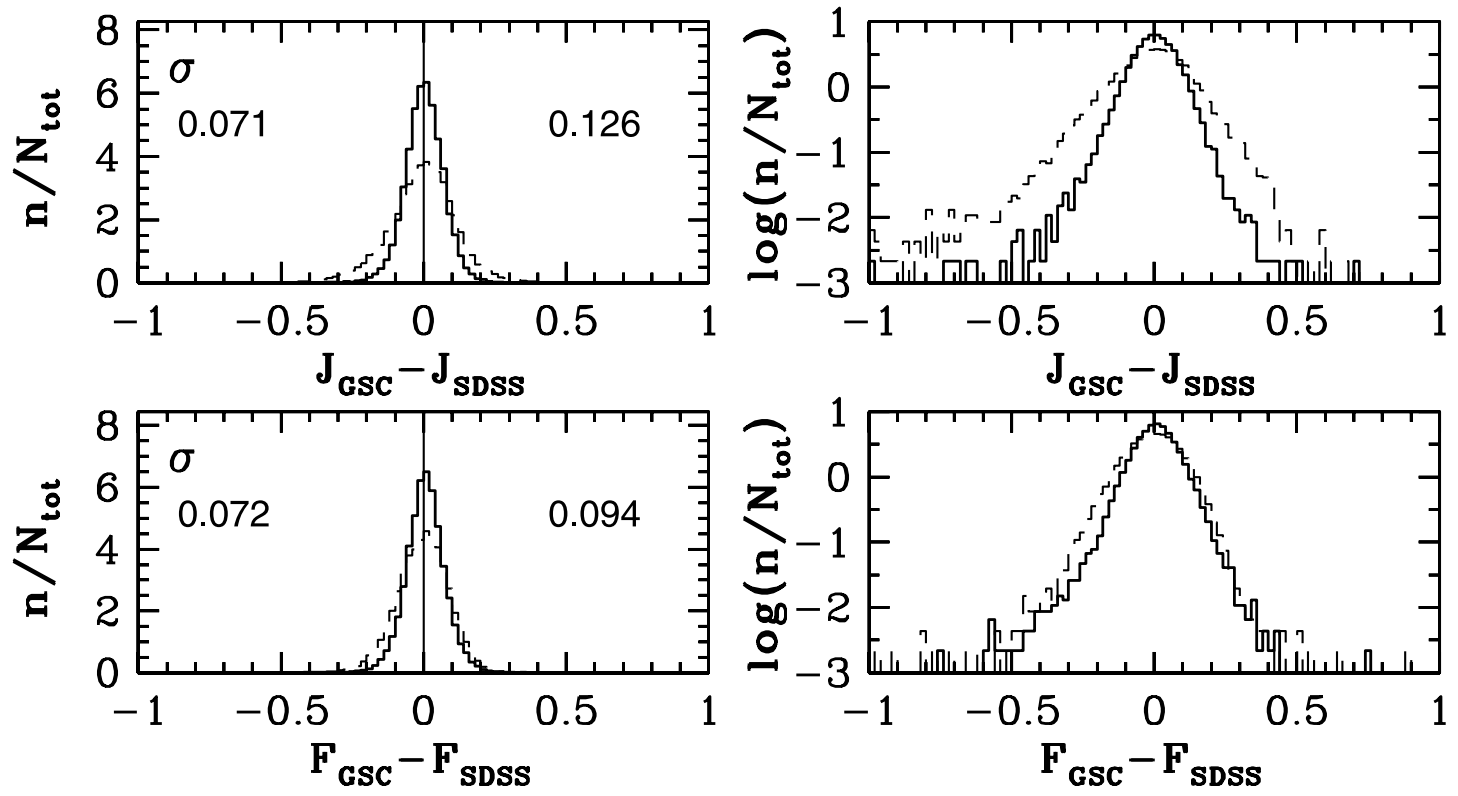

FIG. 11.-Same as Fig. 5, except for the GSC2.2 catalog. [See the electronic edition of the Journal for a color version of this figure.]
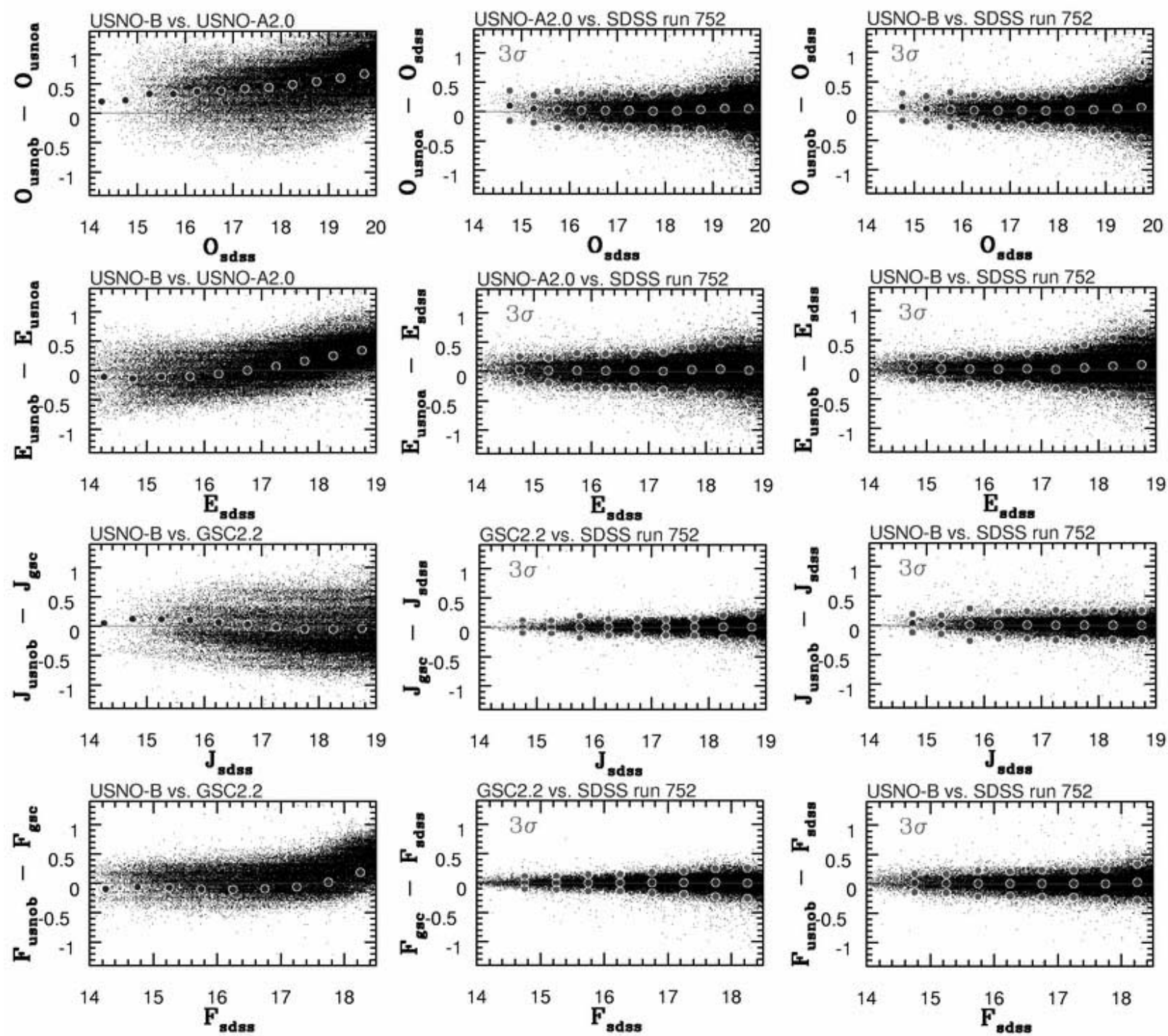

FIG. 12.- Summary of recalibration results for different POSS catalogs, as marked. The POSS-SDSS comparisons, shown in the middle and right panels, are based on the recalibrated magnitudes, while the POSS-POSS comparisons for different input catalogs (left panels) are based on their original magnitudes. Note how the same bands have original magnitudes differing from one catalog to another by as much as $0.5 \mathrm{mag}$. [See the electronic edition of the Journal for a color version of this figure.] 
TABLE 3

The Number of Selected Candidate Variables

\begin{tabular}{|c|c|c|c|c|c|c|}
\hline \multirow[b]{2}{*}{$\begin{array}{l}\text { BAND } \\
\text { (1) }\end{array}$} & \multirow[b]{2}{*}{$\begin{array}{c}m_{\text {faint }} \\
\text { (2) }\end{array}$} & \multicolumn{2}{|c|}{$\chi>3.0$} & \multicolumn{2}{|c|}{$\chi>3.5$} & \multirow[b]{2}{*}{$\begin{array}{l}N_{\text {tot }} \\
\text { (7) }\end{array}$} \\
\hline & & $\begin{array}{c}N_{\text {sel }} \\
\text { (3) }\end{array}$ & $\begin{array}{c}\text { Percent } \\
\text { (4) }\end{array}$ & $\begin{array}{c}N_{\text {sel }} \\
\text { (5) }\end{array}$ & $\begin{array}{c}\text { Percent } \\
\text { (6) }\end{array}$ & \\
\hline$O$ & 19.5 & 11291 & 1.03 & 6436 & 0.59 & $1,087,438$ \\
\hline$E$ & 18.5 & 11246 & 0.95 & 6413 & 0.54 & $1,181,929$ \\
\hline G & 19.5 & 4945 & 0.45 & 3399 & 0.31 & $1,113,771$ \\
\hline$R$ & 18.5 & 6545 & 0.58 & 3624 & 0.32 & $1,124,272$ \\
\hline$I \ldots \ldots \ldots \ldots \ldots \ldots \ldots$ & 17.0 & 3676 & 0.69 & 1701 & 0.32 & 535,839 \\
\hline
\end{tabular}

Notes.-The minimum required magnitude change for the $O$ and $E$ bands is $\Delta m_{\min }=0.3$, and it is $\Delta m_{\min }=0.25$ for the $G, R$, and $I$ bands. Col. (2): The adopted faint limit (using recalibrated POSS magnitudes). Cols. (3) and (4): The number and percentage of selected candidate variables with $\chi>3.0$. Cols. (5) and (6): The number and percentage of selected candidate variables with $\chi>3.5$. Col. (7): The total number of objects brighter than $m_{\text {faint }}$.

residual dependence on magnitude and are significantly better before recalibration, as shown in Figures 8 and 9. Results after recalibration are comparable with those of USNO-B, although slightly better. The main difference between the recalibration of GSC2.2 and other catalogs is the lack of plate information, ${ }^{17}$ which prevented the second recalibration step. Nevertheless, Figures 10 and 11 show that for the GSC2.2 catalog the magnitude dependence of the difference between the SDSS and recalibrated POSS magnitudes is minor.

\subsubsection{Summary of Recalibration Results}

The rms values of the magnitude differences before and after the recalibration for all the catalogs and bands are listed in Table 2 . The final errors for the POSS II magnitudes are generally smaller ( $\sim 0.10 \mathrm{mag}$ ) than for the POSS I magnitudes ( $\sim .15 \mathrm{mag})$, both evaluated for stars brighter than $g=19$. The smallest final errors are obtained with the GSC2.2 catalog, for which they approach 0.07 mag at the bright end. A summary comparison of the original and recalibrated magnitudes for different catalogs is shown in Figure 12. It should be noted that even when the photographic bands are similar (i.e., the USNO-B and GSC2.2 $J$ and $F$ bands), their values can be different by as much as $0.5 \mathrm{mag}$. This clearly shows that without proper recalibration it would be difficult to use data from different catalogs for variability studies.

\section{PRELIMINARY ANALYSIS OF THE POSS-SDSS CATALOGS OF VARIABLE SOURCES}

The comparison of the SDSS photometric catalog with photometrically recalibrated POSS catalogs can yield a large number of variable sources. Various methods can be employed to produce such a list of candidate variables, depending on whether each band/catalog is considered separately or not, on the cutoff values for magnitude differences, on the sample faint limit, etc. The DPOSS catalog is the main catalog used in the subsequent analysis (while the smallest final errors are produced with the GSC2.2 catalog, its public version is not as deep as the DPOSS catalog). For the POSS I survey we chose the USNO-B1.0 catalog (the other option is USNO-A2.0; the photometric errors after recalibration are similar for both USNO catalogs) because it has a more extensive set of astrometric and photometric parameters (proper motions, epochs, etc.). The criteria for selecting can-

\footnotetext{
${ }^{17}$ Observations listed in the catalog are collected from multiple plates, even if confined to a small sky region, and the plate number from which a particular entry was derived is not provided.
}

didate variable sources are described in $\S 3.1$, and a series of tests for estimating the selection reliability are described in $\S 3.2$.

\subsection{Selection Criteria}

When selecting candidate variable sources we consider each POSS band individually for two reasons. First, sometimes POSS observations of the same sky regions were not obtained at the same time, and treating each epoch separately increases the selection completeness for sources variable on short timescales. Second, additional constraints that combine different bands (e.g., "a source must vary in both the $O$ and $E$ bands") can be easily imposed after the initial single-band-based selection. We consider only isolated point sources detected by both POSS and SDSS. The SDSS catalogs have a high completeness $(99.3 \%$ for the magnitude range overlapping POSS surveys; Ivezić et al. 2001), and given that the SDSS is considerably deeper than POSS, the exclusion of orphaned sources has no significant consequence for the samples discussed in $\S \S 4$ and 5 .

For each catalog and band we define the faint magnitude limit $m_{\text {faint }}$, minimum flux variation $\Delta m_{\min }$, and the variation's minimum significance $\chi=\Delta m / \sigma$, where $\Delta m=\left|m_{\mathrm{SDSS}}-m_{\mathrm{POSS}}\right|$ and $\sigma$ is the photometric error. In addition, to be considered for further analysis, objects must pass certain plate quality cuts described in detail in Appendix B (a cumulative quality flag goodPhoto set to 1). The adopted values of selection parameters for each catalog are listed in Table 3, as well as the number of selected candidate variable sources. In general, the $\chi$-condition controls the selection of variable sources at the faint end, and $\Delta m_{\min }$ controls the selection at the bright end. We find that typically $15 \%-20 \%$ of selected candidates simultaneously satisfy conditions in two bands from a given catalog.

These particular selection criteria were adopted after a trialand-error procedure that utilized tests described in $\S 3.2$. We chose to err on the conservative side and increase catalog robustness at the expense of its completeness, since the small number of epochs already introduces substantial incompleteness. Hence, the fraction of variable sources reported here is only a lower limit.

\subsection{Tests of the Selection Reliability}

Given the selection criteria described in $\S 3.1$, it is necessary for subsequent analysis to estimate the completeness and efficiency of the resulting samples. The selection completeness, or the fraction of true variable sources in the analyzed sky region and observed magnitude range selected by the algorithm, 

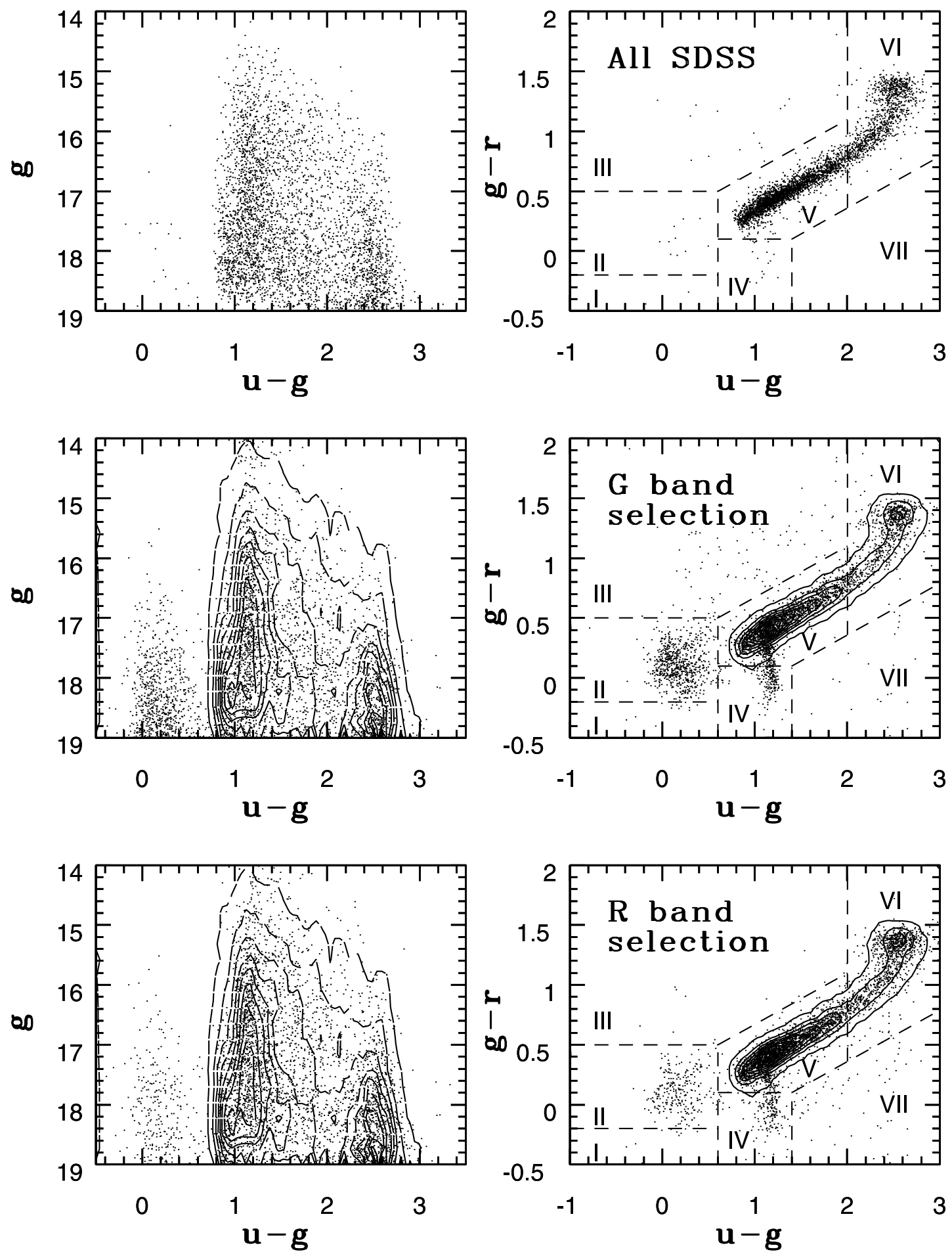

FIG. 13.-Distribution of SDSS-DPOSS candidate variable sources with $g<19$ in representative SDSS color-magnitude (left) and color-color diagrams $(r i g h t)$. The top panels are shown for reference and display a sample of SDSS point sources with the same flux limit and with the same total number of sources ( 23000$)$. The middle and bottom panels display the distributions from the top panels as contours, and variable sources selected from the DPOSS catalog as dots (see entries in Table 3). The regions marked in the right panels are used for quantitative comparison of the overall and variable source distributions (see Table 4). [See the electronic edition of the Journal for a color version of this figure.]

is certainly low because the selection is based on variations in only one bandpass and a fairly large $\Delta m_{\text {min }}$ cutoff compared to the typical amplitudes of variable sources (e.g., most RR Lyrae stars and quasars have peak-to-peak amplitudes $\lesssim 1 \mathrm{mag}$ ). For example, Ivezić et al. (2000) used two-epoch SDSS measurements to select candidate RR Lyrae stars and obtained a completeness of $\sim 50 \%$ for a $\Delta m_{\text {min }}$ cutoff of $0.15 \mathrm{mag}$. With larger $\Delta m_{\text {min }}$ cutoffs and the conservative $\chi$ adopted here, the ex- pected selection completeness for RR Lyrae stars is about $25 \%$ (see $\S 4$ for a direct measurement). The completeness for other types of variable source depends on the shapes and amplitudes of their light curves and is hard to estimate, but for most sources is similarly low. While such a low completeness cannot be avoided with the available data, its stability across the sky can be controlled. This stability is demonstrated by the lack of features in the distribution of quasars selected by variability, as well as by 
TABLE 4

The Distribution of Variable Sources in the $g-r$ versus $u-g$ Diagram

\begin{tabular}{|c|c|c|c|c|c|c|c|}
\hline \multirow[b]{2}{*}{$\begin{array}{l}\text { REGION } \\
\text { (1) }\end{array}$} & \multirow[b]{2}{*}{$\begin{array}{l}\text { NAME } \\
(2)\end{array}$} & \multicolumn{3}{|c|}{$G$ BAND } & \multicolumn{3}{|c|}{$R$ B $\mathrm{AND}$} \\
\hline & & $\begin{array}{l}\text { All } \\
(\%) \\
(3)\end{array}$ & $\begin{array}{c}\text { Variable } \\
(\%) \\
(4)\end{array}$ & $\begin{array}{c}\text { Variable/All } \\
\text { (5) }\end{array}$ & $\begin{array}{l}\text { All } \\
(\%) \\
(6)\end{array}$ & $\begin{array}{c}\text { Variable } \\
(\%) \\
(7)\end{array}$ & $\begin{array}{c}\text { Variable/All } \\
(8)\end{array}$ \\
\hline I & White dwarfs & 0.15 & 0.41 & 2.69 & 0.05 & 0.30 & 5.56 \\
\hline II ....................... & Low-z QSOs & 0.60 & 20.39 & 33.96 & 0.27 & 7.06 & 26.62 \\
\hline III......................... & Binary stars & 0.11 & 4.18 & 38.20 & 0.10 & 1.08 & 10.27 \\
\hline 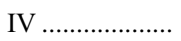 & RR Lyrae stars & 0.60 & 6.68 & 11.04 & 0.44 & 5.49 & 12.34 \\
\hline V & Blue stars & 69.09 & 47.10 & 0.68 & 67.68 & 56.65 & 0.84 \\
\hline VI ......................... & Red stars & 29.43 & 21.62 & 0.73 & 31.42 & 27.62 & 0.88 \\
\hline VII ....................... & High- $z$ QSOs & 0.05 & 1.59 & 34.63 & 0.05 & 2.54 & 51.43 \\
\hline
\end{tabular}

Notes.-Col. (1): The regions whose boundaries are shown in Fig. 13. Col. (2): An approximate description of the dominant source type. Col. (3): Fraction of all $G$-selected SDSS sources in the region (based on Table 3). Col. (4): Fraction of $G$-selected candidate SDSS-DPOSS variable sources in the region, with the $\Delta m_{\min }=0.25$ and $\chi=3.5$ selection from Table 3. Col. (5): Ratio of percentages of variable and all $G$-selected objects in the region. Col. (6): Fraction of all $R$-selected SDSS sources in the region (based on Table 3). Col. (7): Fraction of $R$-selected candidate SDSS-DPOSS variable sources in the region, with the $\Delta m_{\min }=0.25$ and $\chi=3.5$ selection from Table 3. Col. (8): Ratio of percentages of variable and all $R$-selected objects in the region.

the recovery of known structures in the distribution of RR Lyrae stars, as discussed in $\S 4.2$.

The selection efficiency, or the fraction of true variable sources in the selected sample, may severely impact the analysis if not sufficiently large. We demonstrate using a series of tests that the selection efficiency is indeed very large (75\%) and thus allows a robust analysis of variable faint optical sources.

The main diagnostic for the robustness of the adopted selection criteria is the distribution of selected candidates in SDSS color-magnitude and color-color space. Were the selection a random process, the selected candidates would have the same distribution as the whole sample. However, we find that the samples of candidate variables have a significantly different distribution, as detailed in $\S 3.2 .1$. The most robust and quantitative test for estimating selection reliability is a comparison with repeated SDSS imaging observations, although applicable to only a small fraction $(\sim 10 \%)$ of the sky area discussed here where such SDSS observations exist. Another powerful test for candidates with large suspected flux variation $(\gtrsim 0.5 \mathrm{mag})$ is a simple visual comparison of POSS and SDSS images. While we found a number of spurious candidates using this method, their fraction is not large enough to significantly affect our results.

\subsubsection{The Distribution of Candidate Variable Sources in SDSS Color-Color Diagrams}

The position of a source in SDSS color-magnitude and colorcolor diagrams is a good proxy for its classification. The distribution of selected candidate variables with $g<19$ (using the DPOSS catalog and $\chi=3.5$ selection criterion) in representative diagrams is shown in Figure 13 (the measured magnitudes are corrected for interstellar extinction using the map from Schlegel et al. [1998]). The top row is shown for reference and displays a sample of randomly selected SDSS point sources with the same flux limits as used for selecting variable sources. The middle and bottom rows compare the distributions of this reference sample, shown as contours, to the distributions of candidate variable sources, shown as dots.

The distributions of candidate variable sources and those of the reference sample are different, demonstrating that the candidate variables are not randomly selected from the whole sample. The most obvious difference between the distributions is a much higher fraction of quasars (recognized by their UV excess, $u-g<0.6$ ) in the variable sample (quasars are known to be variable on the long timescales discussed here; see $\S 5$ ). Another notable difference is the presence of RR Lyrae stars $(u-g \sim$ $1.2, g-r \sim 0$ ) among the candidate variables. Thus, known variable sources indeed dominate the selected candidates.

In order to quantify these differences, as well as those in other parts of the color-color diagram, we divide color-color diagrams into seven characteristic regions, each dominated by a particular type of source (for more details about the distribution of point sources in SDSS color-color diagrams, see Lenz et al. [1998], Fan [1999], Finlator et al. [2000], and Richards et al. [2002]). The fractions of variable and all sources in each region are listed in Table 4. Notably, the fraction of variable sources that are found in region II, representative of numerous low-redshift quasars, is $\sim 34$ times higher than for the reference sample. ${ }^{18}$ The corresponding fraction for region VII (which includes high-redshift quasars and, possibly, variable stars) is about the same ( $\sim 34)$, but the statistics are less robust due to a smaller number of sources. Another quantitative representation of the color differences introduced by the variability requirement is shown for $u-g$ color in Figure 14. These differences and the results obtained in $\S 3.2 .2$ demonstrate that the sample of selected candidate variables is not dominated by spurious objects.

The fraction of selected candidate variables across the sky is stable and, in particular, does not depend on the stellar number density, nor does it show jumps at the boundaries of the Schmidt plates. Figure 15 illustrates this stability for a $2^{\circ} .5$ wide strip centered on the celestial equator, where the fraction of candidate variables remains $\sim 0.8 \%$ (not corrected for unknown selection incompleteness), despite the stellar counts varying by a factor of $\sim 3$ (a slight increase at $\alpha \sim 230$ is caused by RR Lyrae in the Sgr dwarf tidal stream; see $\S 4$ ). A similarly stable behavior is observed when color-selected quasars are further subselected by variability.

\subsubsection{The Comparison with Repeated SDSS Imaging Observations}

The analysis presented in $\S 3.2 .1$ shows that the selected candidate variables are not dominated by spurious sources. Here we obtain a quantitative estimate of the selection efficiency using repeated SDSS imaging data. For about $10 \%$ of the sky

\footnotetext{
${ }^{18}$ The fraction of low-redshift quasars is higher for blue selection because the DPOSS $m_{\text {faint }}$ cutoff is brighter in the red band (see Table 3).
} 

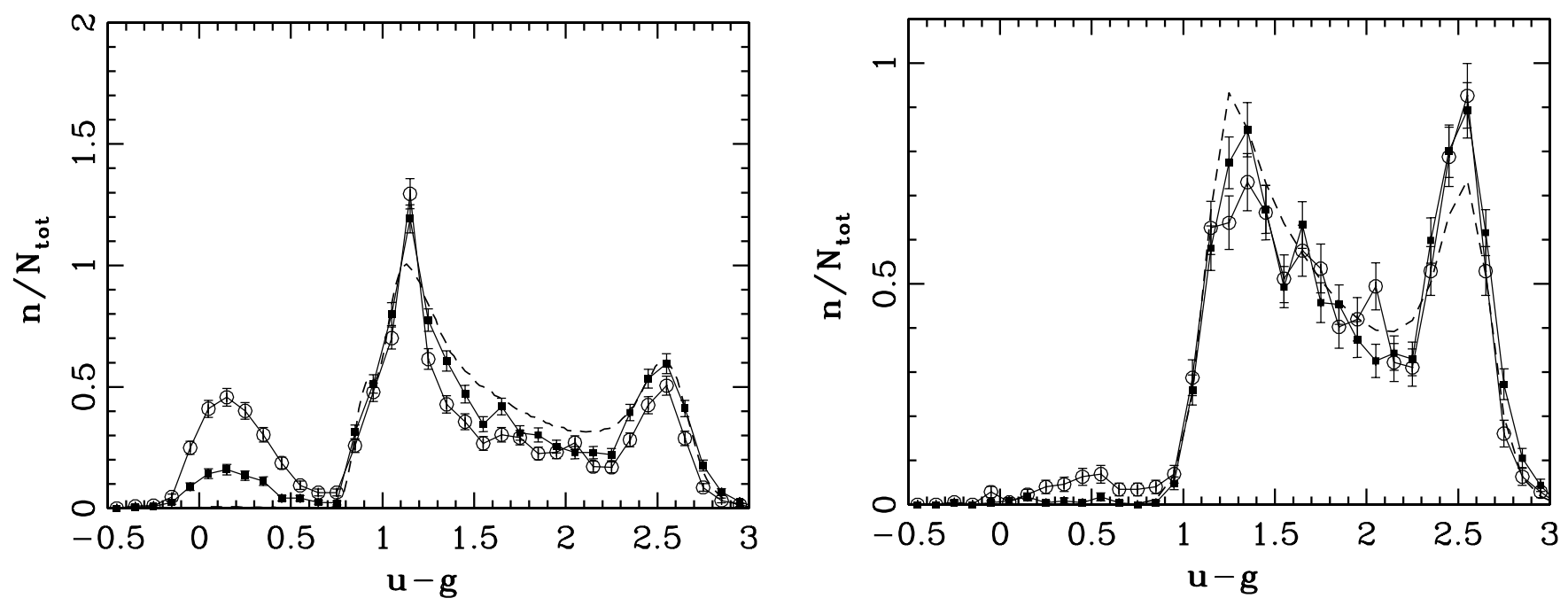

FIG. 14.-Comparison of $u-g$ probability density distributions (i.e., the integrals of the plotted curves are 1 by definition) for SDSS-DPOSS candidate variable sources (circles, $G$ selection; squares, $R$ selection), and for a reference sample with the same magnitude limit (dashed line). The left panel shows all sources, and the right panel shows a subset with $g-r>0.4$ (designed to avoid the majority of low-redshift quasars; see Fig. 13). The peak at $u-g \sim 0.2$ is dominated by quasars, the peak at $u-g \sim 1.15$ by RR Lyrae stars, and the peak at $u-g \sim 2.5$ by M stars. [See the electronic edition of the Journal for a color version of this figure.]

area analyzed here (the SDSS southern equatorial strip; see York et al. 2000), there exist between six and nine epochs of SDSS imaging, obtained over a period of 4 years. Due to both a larger number of epochs and more accurate photometry $(\sim 0.02 \mathrm{mag}$; for details see Ivezić et al. 2003), these data have a much higher completeness and efficiency for discovering variable sources than the SDSS-POSS data presented here. We select variable sources from repeated SDSS scans by requiring a minimum variability larger than $0.075 \mathrm{mag}$ in the $g$ band. This selection results in a negligible fraction of spurious candidates $(<1 \%$, determined using SDSS data obtained $3 \mathrm{hr}$ apart) and a high completeness (for example, more than $90 \%$ for RR Lyrae stars), and is also sensitive to long-period variables and quasar vari-

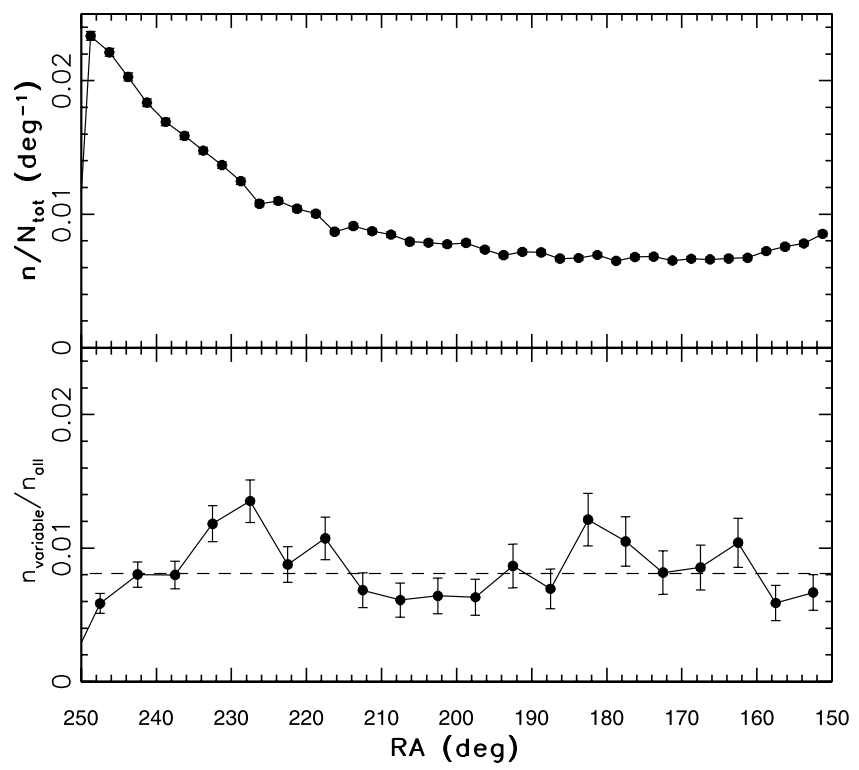

FIG. 15.-Counts of all sources (top) and the fraction of candidate variables in a 2.5 wide strip centered on the celestial equator, as a function of right ascension. The counts increase by a factor of $\sim 3$ toward the left edge because of the decreasing Galactic latitude. The fraction of candidate variables stays constant (at $0.8 \%$ ) within Poissonian noise. [See the electronic edition of the Journal for a color version of this figure.] ability (Ivezić et al. 2003). About 7\% of point sources brighter than $g=19$ pass the adopted selection cut.

Using the SDSS-DPOSS $G$-band candidates selected by the $\Delta m_{\min }=0.25, \chi=3.5$ criteria (also see Table 3 ), we find that repeated SDSS scans (at least six epochs) exist for 102 sources. About $75 \%$ of these ( 76 objects) are confirmed as variable by SDSS data. The distributions of confirmed and spurious SDSSDPOSS variables in SDSS color-color diagrams are compared in Figure 16. It is not surprising that most of the spurious SDSSDPOSS variables are found in the stellar locus, because for a given contamination fraction (which is not expected to be a strong function of color) most of the contaminants come from the most populated part of the diagram. Repeating this analysis separately for sources from inside and outside the stellar locus, we find that the fraction of true variable sources among the selected candidates from the locus is $52 \%$, while outside the locus it is as high as $94 \%$. Assuming that no more than $10 \%$ of sources from the locus are truly variable, the former fraction implies that the decision to tag a source as a candidate variable is correct in more than $95 \%$ of cases (for $g<19$ ).

\subsubsection{The Large-Amplitude Variables and Visual Comparison of Images}

The presumed large-amplitude variables ( $\gtrsim 1 \mathrm{mag}$ ) may be more likely to be spurious (e.g., due to various defects on photographic plates). This possibility cannot be robustly tested using methods from $\S 3.2 .2$ due to insufficient number of sources. On the other hand, presumed variations with such large amplitudes can be tested by the visual comparison of SDSS and POSS images. The distribution of 76 SDSS-DPOSS candidates with $0.7<\Delta G<1$ and 36 candidates with $1<\Delta G<3$ in SDSS color-color diagrams is shown in Figure 17. As is evident, their distribution does not follow the distribution for the reference sample, indicating that they are not dominated by spurious candidates. We have visually inspected POSS and SDSS images for these 112 candidates and found that less than $\sim 30 \%$ may have been affected by nearby bright stars. Additional visual inspection of large-amplitude variables selected using the USNO-A2.0 catalog recovered a spectacular case shown in the top panels of Figure 18. After analyzing the plate print, as well 

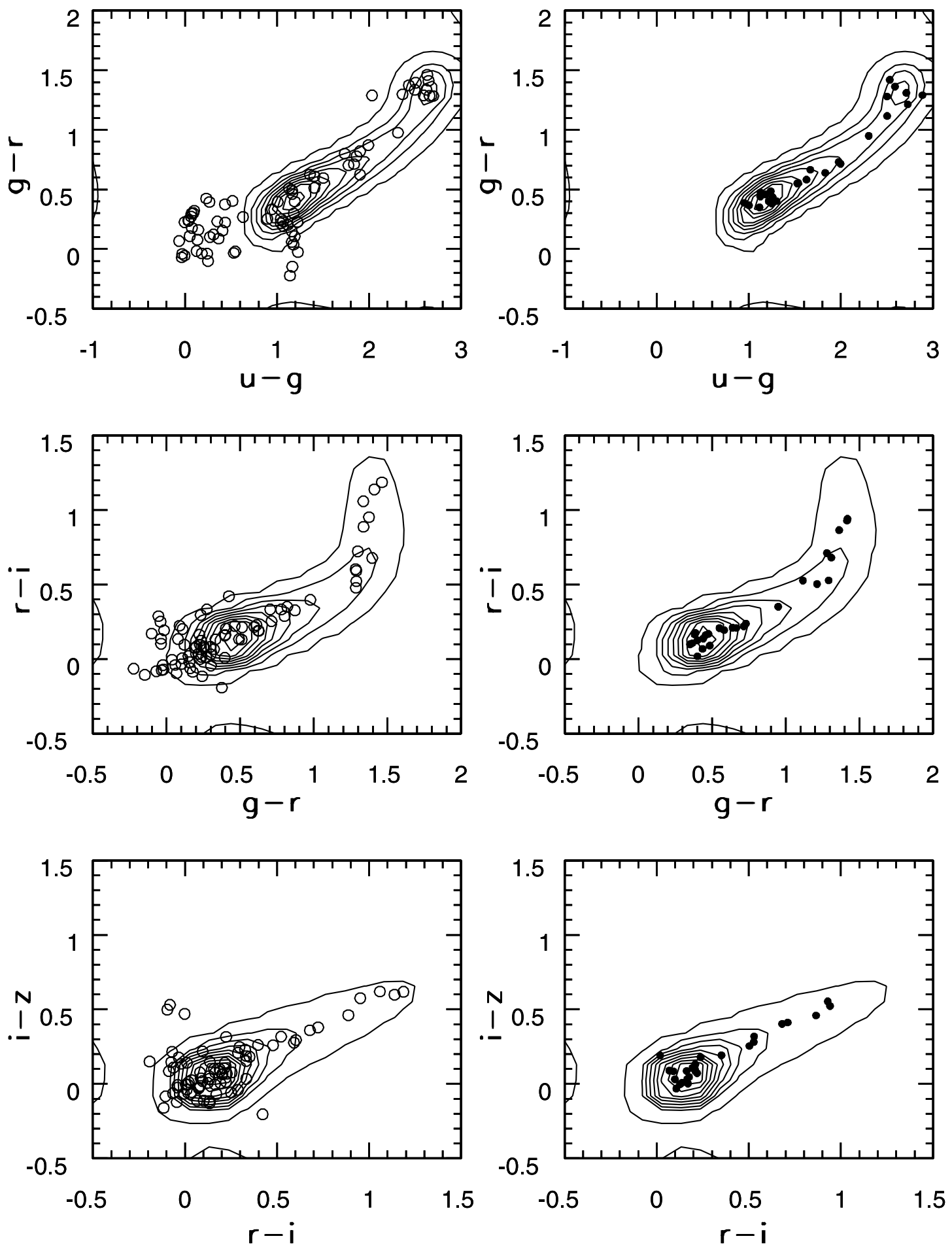

FIG. 16.-Distribution of SDSS-DPOSS candidate variable sources $\left(\Delta m_{\min }=0.25, \chi=3.5 \mathrm{G}\right.$ selection; see Table 3$)$ confirmed as varying by multiepoch SDSS imaging (left panels; $75 \%$ of the sample), and those that did not show any evidence of variability (right panels; $25 \%$ of the sample). Note that the latter are mostly found in the stellar locus. [See the electronic edition of the Journal for a color version of this figure.]

as the brightness profiles, we concluded that the two bright POSS sources were an artifact ${ }^{19}$ ( probably caused by splattered liquid on the POSS plate). While this is a disappointing outcome, it nevertheless vividly demonstrates the ability of the selection method to recognize differences between POSS and

\footnotetext{
${ }^{19}$ This is a good example of benefits afforded by large collaborations: they provide an increased statistical chance of working with a sufficiently senior member familiar with old technologies and all their pitfalls.
}

SDSS data. Another example of a spurious candidate is shown in the bottom panels of Figure 18. Due to a nearby star, which happened to be a large-proper-motion object, the candidate's POSS photometry was noticeably affected, while the more accurate SDSS photometry reported a single object with a correct magnitude.

Despite these pitfalls, the SDSS-POSS comparison does yield true large-amplitude variables. For example, one of the sources with an SDSS-POSS magnitude difference of $\sim 2 \mathrm{mag}$ is in the 

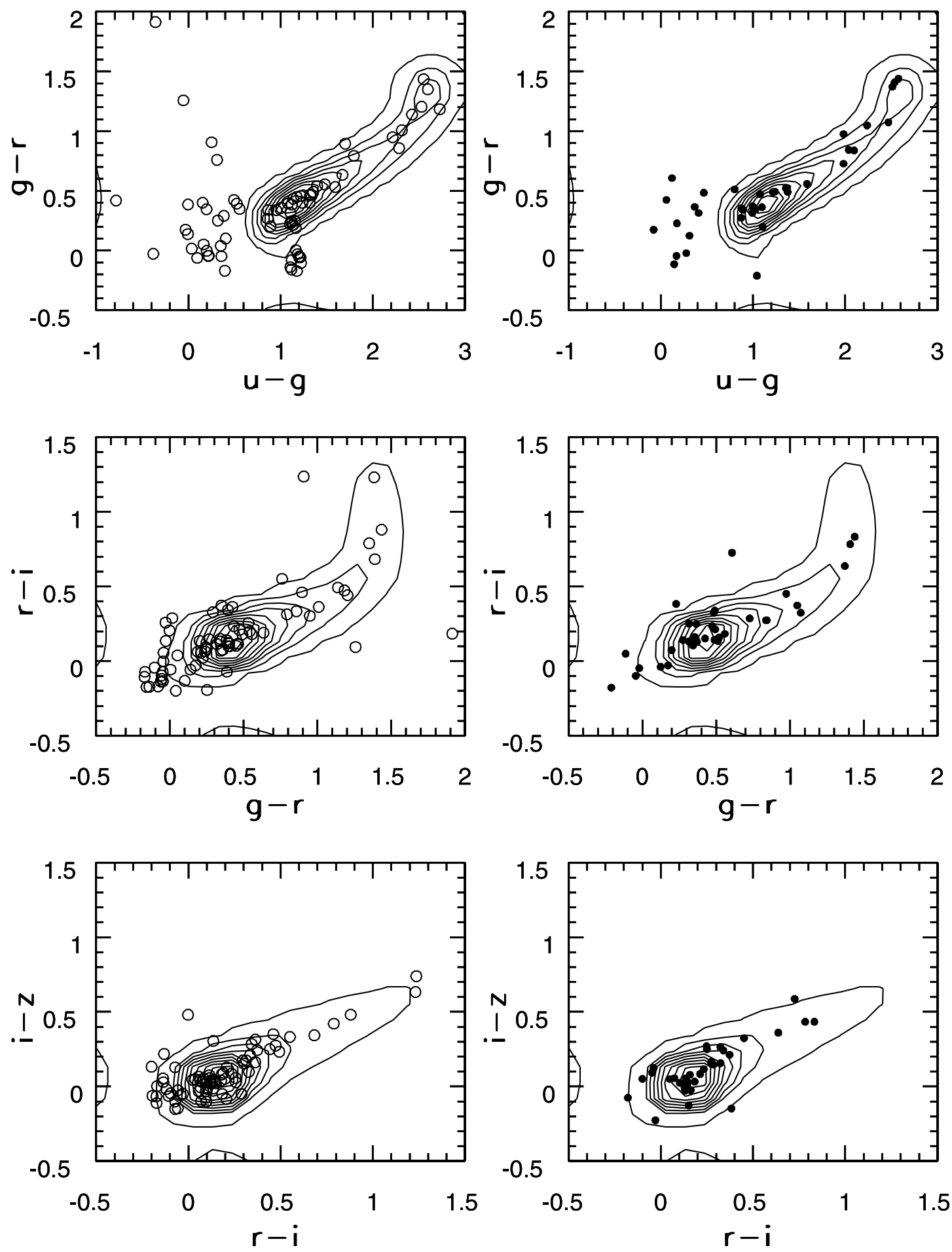

FIG. 17.-Distribution of SDSS-DPOSS candidate variable sources with $g<19$ and large amplitudes in representative SDSS color-color diagrams. The symbols show 76 objects with $0.75<|\Delta G|<1$ in the left panels, and 36 objects with $1<|\Delta G|<3$ in the right panels. The overall distributions of SDSS sources with the same magnitude limit are shown by the contours. [See the electronic edition of the Journal for a color version of this figure.]

region multiply observed by SDSS (12 epochs). The available SDSS data demonstrate that it is a long-period variable with a peak-to-peak amplitude exceeding $5 \mathrm{mag}$. This star, and another example of a large-amplitude variable, are shown in Figure 19.

\section{THE MILKY WAY HALO STRUCTURE TRACED BY CANDIDATE RR LYRAE STARS}

As recently shown (Ivezić et al. 2000, 2004c, 2004d; Vivas et al. 2001), faint RR Lyrae stars have a very clumpy distribu- tion on the sky (most prominent features are associated with the Sgr dwarf tidal stream). This substructure offers a test of the spatial homogeneity of the selection algorithm: the known clumps ought to be recovered to some extent by the candidate RR Lyrae stars selected here, if the selection algorithm is robust. Furthermore, if such robustness can be demonstrated, the SDSSPOSS candidates can be utilized to quantify the halo substructure in the areas of sky for which multiepoch SDSS data do not exist. 

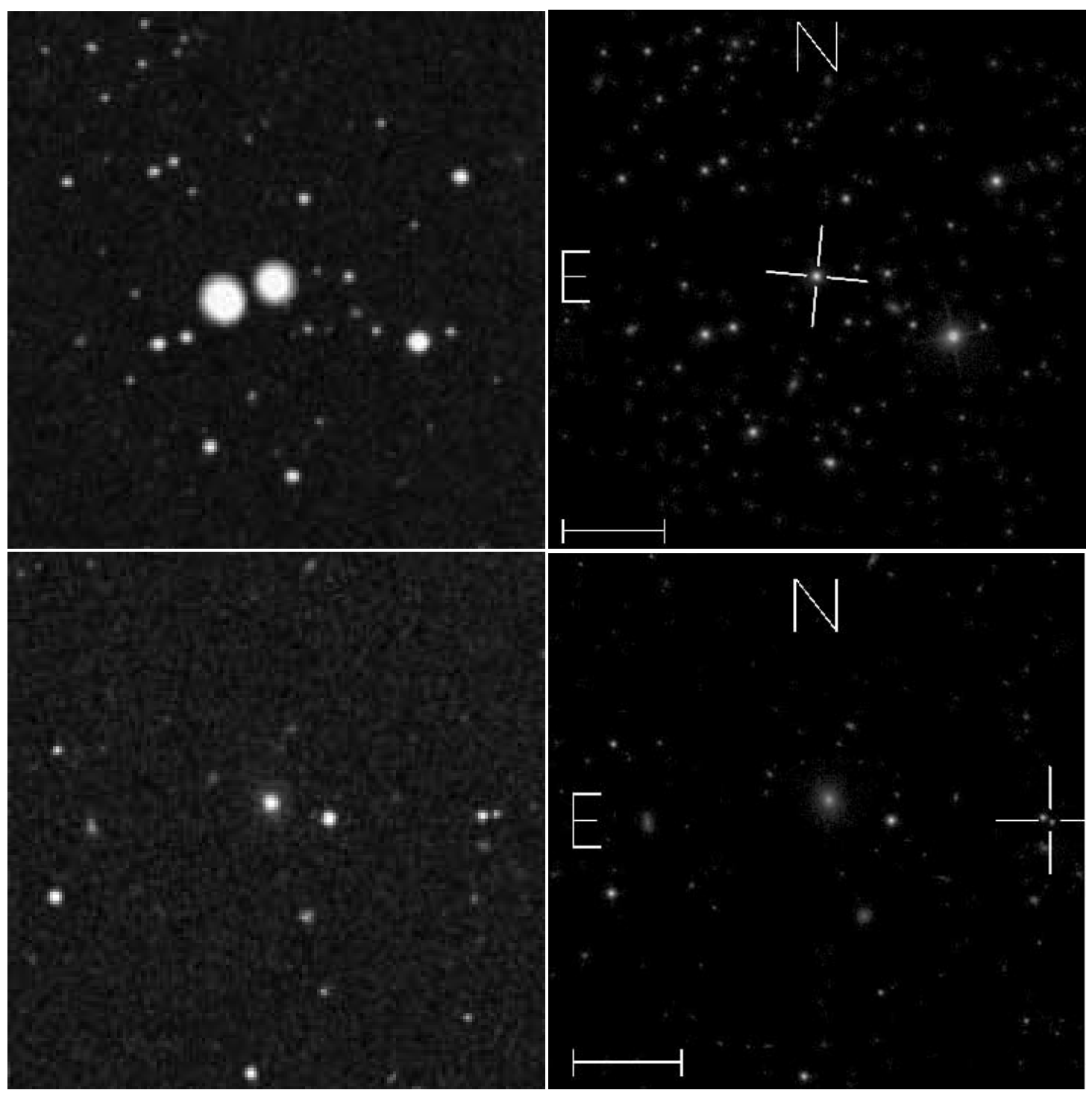

FIG. 18.-Examples of spurious candidate variable sources. The right panels display the $5^{\prime} \times 5^{\prime} g$-band SDSS images, and the left panels display the blue POSS I images on the same scale and with the same orientation. In the top panels, the source marked by a cross was selected as a large-amplitude candidate variable. The visual inspection of the POSS image confirmed that a much brighter source existed in the POSS image, as well as another nearby bright source, both of which turned out to be artifacts. The bottom panels show an example in which the POSS photometry was noticeably affected by a nearby source (which happened to be a large-proper-motion object).

\subsection{The $u-g$ Color Distribution of Candidate RR Lyrae Stars}

Before proceeding with the analysis of spatial distributions of candidate RR Lyrae stars, we test their selection robustness using a method introduced ${ }^{20}$ by Ivezić et al. (2000). RR Lyrae stars have somewhat redder $u-g$ color $(\sim 0.2 \mathrm{mag})$ than stars with similar effective temperature (i.e., $g-r$ color) that are not on the horizontal giant branch. Since the $u$-band flux is not used in the selection of variable objects (all POSS bands are redder than the SDSS $u$ band), this offset is a robust indication that the candidate variables are dominated by true RR Lyrae stars. Figure 20 compares the $u-g$ color distribution for candidate variable objects to the distribution for all sources in a narrow $g-r$ range $(0-0.05$, designed to exclude the main stellar locus). As is evident, the selected candidates have redder $u-g$ color than the full sample, in agreement with the color distri-

\footnotetext{
${ }^{20}$ This method was suggested to Ivezić et al. by the referee A. Saha.
}

bution of RR Lyrae stars selected using light curves obtained by the QUEST survey (for details see Ivezić et al. 2005). The difference is more pronounced for the selection in blue bands (because the variability amplitude decreases with wavelength), and somewhat more pronounced for the GSC catalog than for the DPOSS catalog. The counts of selected candidates are consistent with the conclusion from $\S 3$ that the decision to tag a source as a candidate variable is correct in more than $95 \%$ of cases.

Completeness and selection efficiency for RR Lyrae stars was determined using a complete sample of 82 RR Lyrae stars discovered by the QUEST survey (isolated subsample with $r<$ 19.5) and discussed by Ivezić et al. (2005). Using the $\chi=3.5$ and $\Delta m_{\min }=0.1$ criteria to tag candidates in the DPOSS $G$ or $R$ band, a total of 26 variable objects were selected, where 21 $(81 \%)$ of these stars are also QUEST RR Lyrae stars, implying a $26 \%$ completeness and $81 \%$ efficiency for the selection of RR Lyrae candidates. This relatively low completeness does not prevent the use of such samples for statistical studies of halo structure because the selection is primarily determined by 


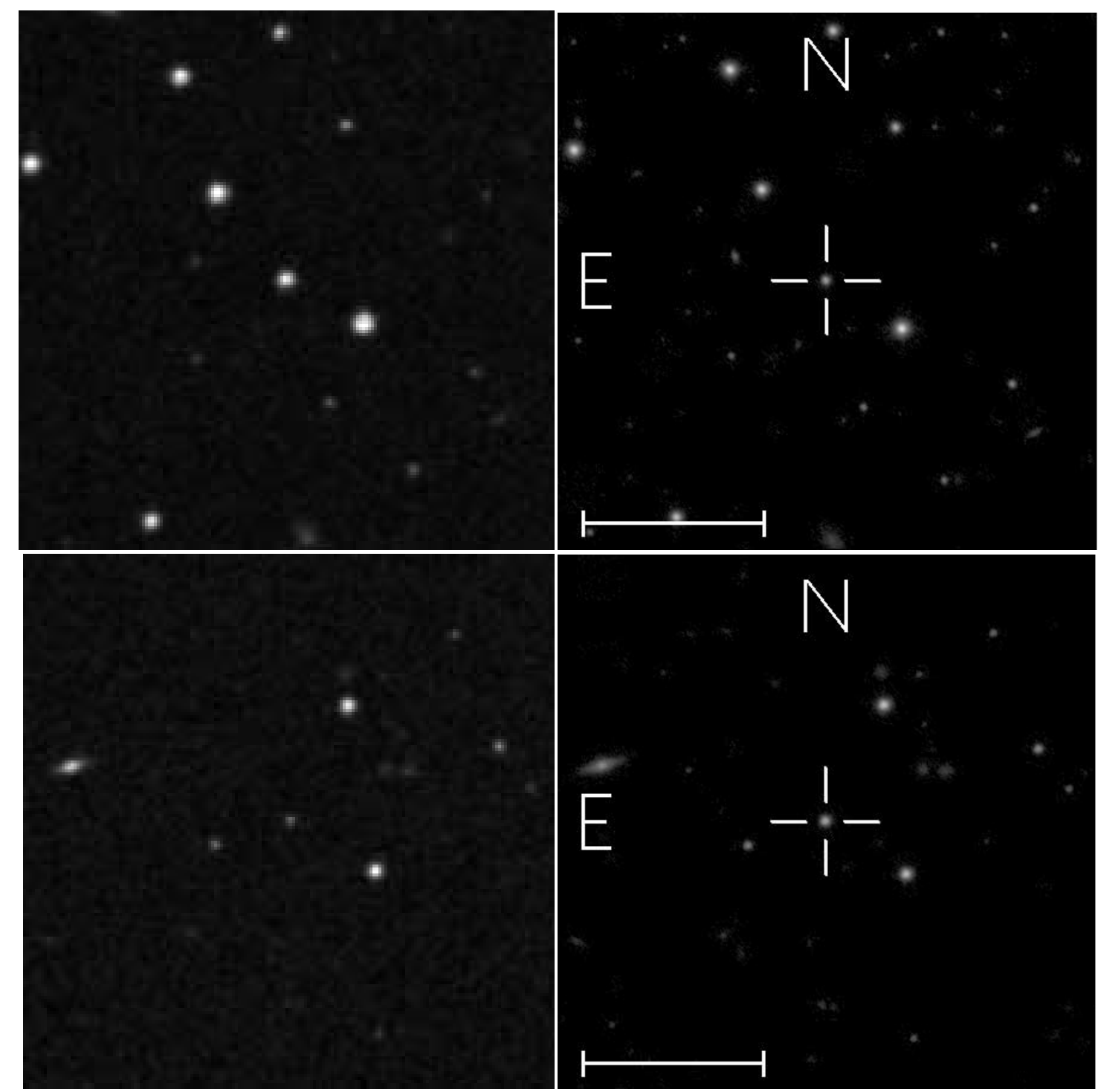

FIG. 19.-Examples of large-amplitude $(\sim 1.5 \mathrm{mag})$ candidate variable sources. The right panels display the $3^{\prime} \times 3^{\prime} g$-band SDSS images, and the left panels display the blue POSS II images on the same scale and with the same orientation. The sources marked by a cross are clearly variable. The top source was brighter in POSS, and the bottom source in SDSS.
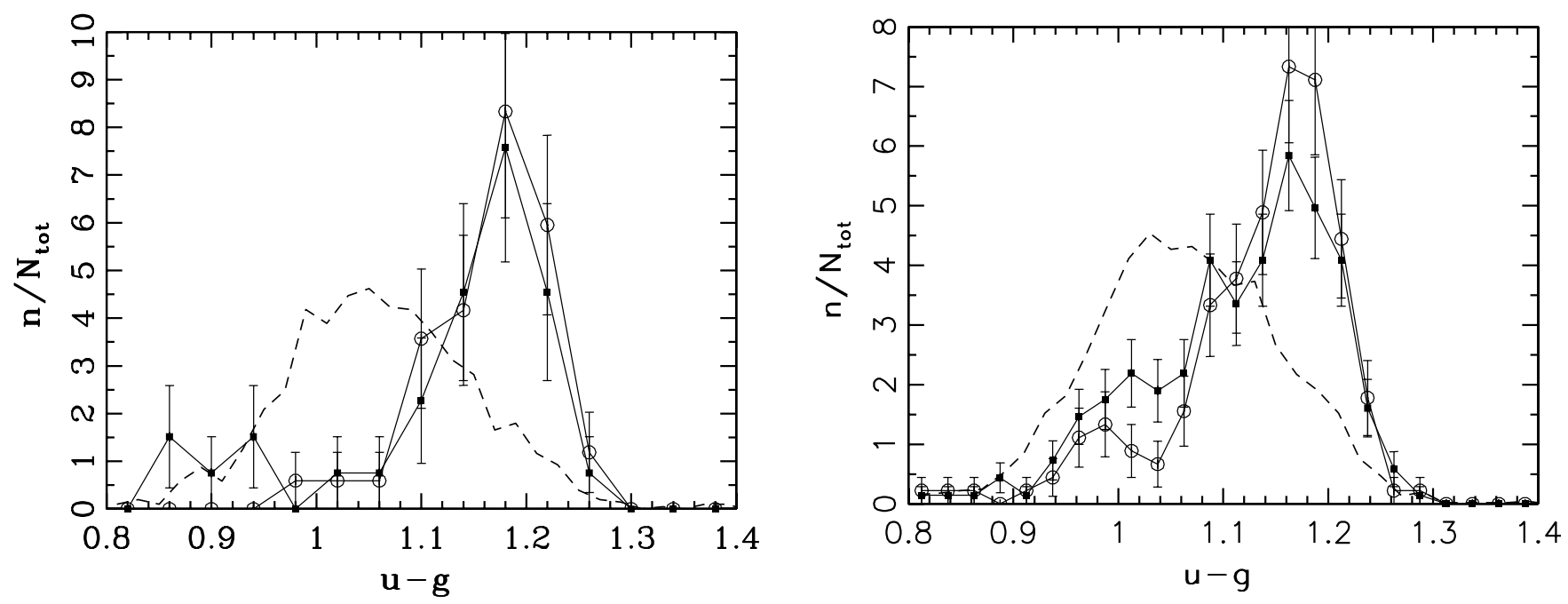

FIG. 20.-Comparison of $u-g$ distributions in the range characteristic for RR Lyrae stars, for candidate variables (symbols with error bars; analogous to Fig. 14) and for a reference sample (dashed line), for sources with $0<g-r<0.05$ and $u<20.5$ (left, DPOSS-based selection; right, GSC-based selection; circles, selection in blue band; squares, selection in red band). Note that variable objects, dominated by RR Lyrae stars, have redder $u-g$ colors than the reference (full) sample. [See the electronic edition of the Journal for a color version of this figure.] 


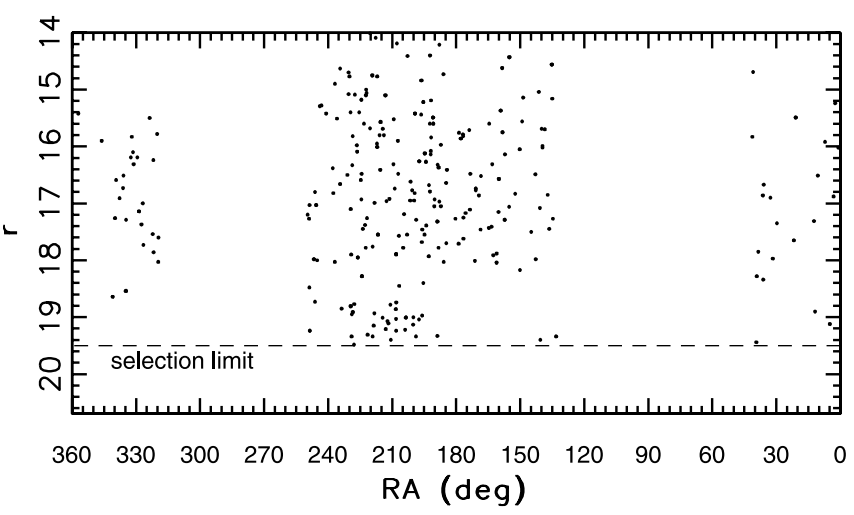

FIG. 21.-Magnitude-position distribution of 258 SDSS-DPOSS RR Lyrae candidates within $5^{\circ}$ of the celestial equator. The sample completeness is fairly uniform for $r<19$ and decreases with $r$ toward the selection faint limit of $r=19.5$. The clumps easily discernible at $(\alpha, r) \sim(210,19.2)$ and $(30,17-18)$ are associated with the Sgr dwarf tidal stream. The clumps at (190, 15.5-16.5) and $(330,17)$ have also been previously reported. The clump at $(\sim 225, \sim 15.5)$ is a new detection. [See the electronic edition of the Journal for a color version of this figure.]

the light-curve phase difference between the two observations and is thus essentially uncorrelated with any relevant physical property.

\subsection{The Spatial Distribution of Candidate RR Lyrae Stars}

Using the above selection criteria (object is tagged if it satisfies criteria in the DPOSS $G$ or $R$ band), we isolate $679 \mathrm{RR}$ Lyrae candidates by adopting color boundaries from Ivezić et al. (2005). The magnitude-position diagram for 258 candidates within $5^{\circ}$ of the celestial equator is shown in Figure 21. The sample completeness is fairly uniform for $r<19$ and decreases with $r$ toward the selection faint limit of $r=19.5$ (corresponding to $\sim 60 \mathrm{kpc})$.

As discussed by Ivezić et al. (2005), an advantage of the data representation utilized in Figure 21 (magnitude-angle diagram) is its simplicity: only "raw" data are shown, without any postprocessing. On the other hand, it can be argued that the identification of overdensities discussed above is somewhat subjective and that their significance is not quantitatively estimated. Furthermore, the magnitude scale is logarithmic, and thus, the spatial extent of structures is heavily distorted. In order to avoid these shortcomings, we have applied a Bayesian method for estimating continuous spatial density distribution developed by Ivezić et al. (see their Appendix B). The resulting spatial density (multiplied by the cube of the galactocentric radius) computed using this method, for data displayed in Figure 21, is shown in the top panel of Figure 23. The advantage of that representation is that it better conveys the significance of various local overdensities. We refer to the various clumps discussed below using their positions in Figures 21 and 22, but we use Figure 23 to confirm their significance.

The clumps easily discernible at $(\alpha, r) \sim(210,19.2)$ and at $(30,17)$ are associated with the Sgr dwarf tidal stream (J2000.0 coordinates are used throughout). The clumps at $(185,16.5)$ and $(330,16.5)$ have also been previously reported (Vivas et al. 2001; Ivezić et al. 2004d). The recovery of these known structures suggests that the clump at $(\sim 225, \sim 15.5)$, which has not been previously reported, is probably a robust detection. Another previously unrecognized clump is detected around $\alpha \sim 240^{\circ}, \delta \sim$ $50^{\circ}$ (see Fig. 22). The significance of these newly recognized structures will be placed in the broader context of other available data elsewhere, when larger samples of other halo tracers, needed

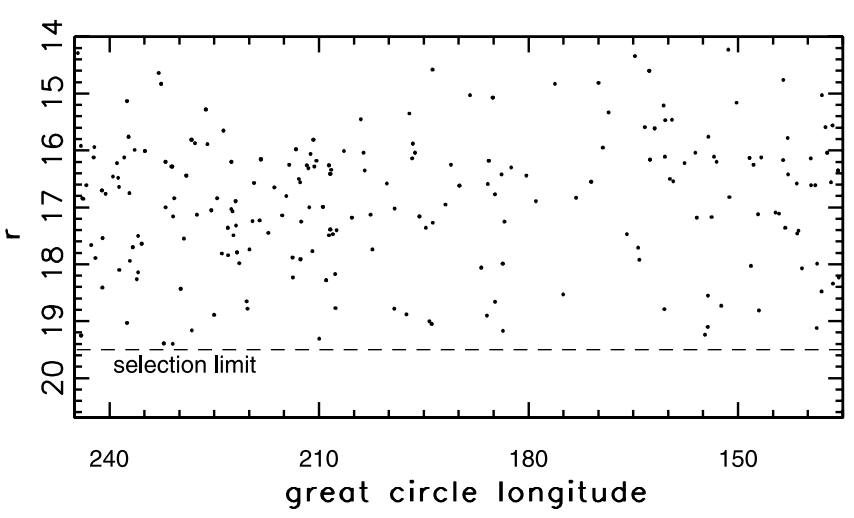

FIG. 22.- Same as Fig. 21, except that the 222 candidates are selected from a $10^{\circ}$ wide strip centered on a great circle defined by a node at $\alpha=95^{\circ}$ and inclination of $65^{\circ}$ (for more details about great-circle coordinates, see Pier et al. 2003). Note the very inhomogeneous structure and, in particular, the fairly prominent feature at the longitudes $210^{\circ}-240^{\circ}\left(\alpha \sim 240^{\circ}\right.$ and $\delta \sim 50^{\circ}$ ), with $r \sim 16-18$. [See the electronic edition of the Journal for a color version of this figure.]

for independent confirmation of the structures, become available (e.g., we are currently comparing these samples to M-giant samples selected using 2MASS [Two Micron All Sky Survey] catalogs).

\section{THE LONG-TERM VARIABILITY OF QUASARS}

The optical continuum variability of quasars has been recognized since their first optical identification (Matthews \& Sandage 1963), and it has been proposed and utilized as an efficient method for their discovery (van den Bergh et al. 1973; Hawkins 1983; Hawkins \& Véron 1995). The observed characteristics of the variability of quasars are frequently used to constrain the origin of their emission (e.g., Kawaguchi et al. 1998 and references therein; Martini \& Schneider 2003).

Recently, significant progress in the description of quasar variability has been made by employing SDSS data (de Vries et al. 2003, 2005, hereafter dV03, dV05; Vanden Berk et al. 2004). The size and quality of the sample analyzed by Vanden Berk et al. (2004; two-epoch photometry for 25,000 spectroscopically confirmed quasars) allowed them to constrain how quasar variability in the rest-frame optical/UV regime depends on the rest-frame time lag, luminosity, rest wavelength, redshift, presence of radio and X-ray emission, and presence of broad absorption line outflows. However, the time lags probed by the available SDSS data (up to $3 \mathrm{yr}$ ) are too short to detect deviations of the structure function (the rms scatter of measured magnitudes; see eq. [1] in dV03) from a simple power law that are expected for long time lags (Cid Fernandes et al. 2000 and references therein).

The much longer time lags between POSS and SDSS ( $\sim 50 \mathrm{yr}$ in the observer's frame) offer the possibility of detecting such deviations, despite larger photometric errors for the POSS catalogs, and of studying the long-term characteristics of quasar variability. Using a recalibration approach similar to the one described here (except that only fields around known quasars were recalibrated), dV03 studied long-term variability for 3791 quasars from the SDSS Early Data Release (Stoughton et al. 2002). While this paper was under peer review, de Vries et al. extended their work and analyzed a sample of $\sim 42,000$ quasars with historic observations from POSS and more recent data from SDSS and the Two Degree Field QSO Redshift Survey (dV05). Here we have used a sample of similar size $(\sim 17,000$; Schneider et al. 2003) but also combined it with the results for short-term quasar variability from Ivezić et al. (2004b, hereafter I04). The large 

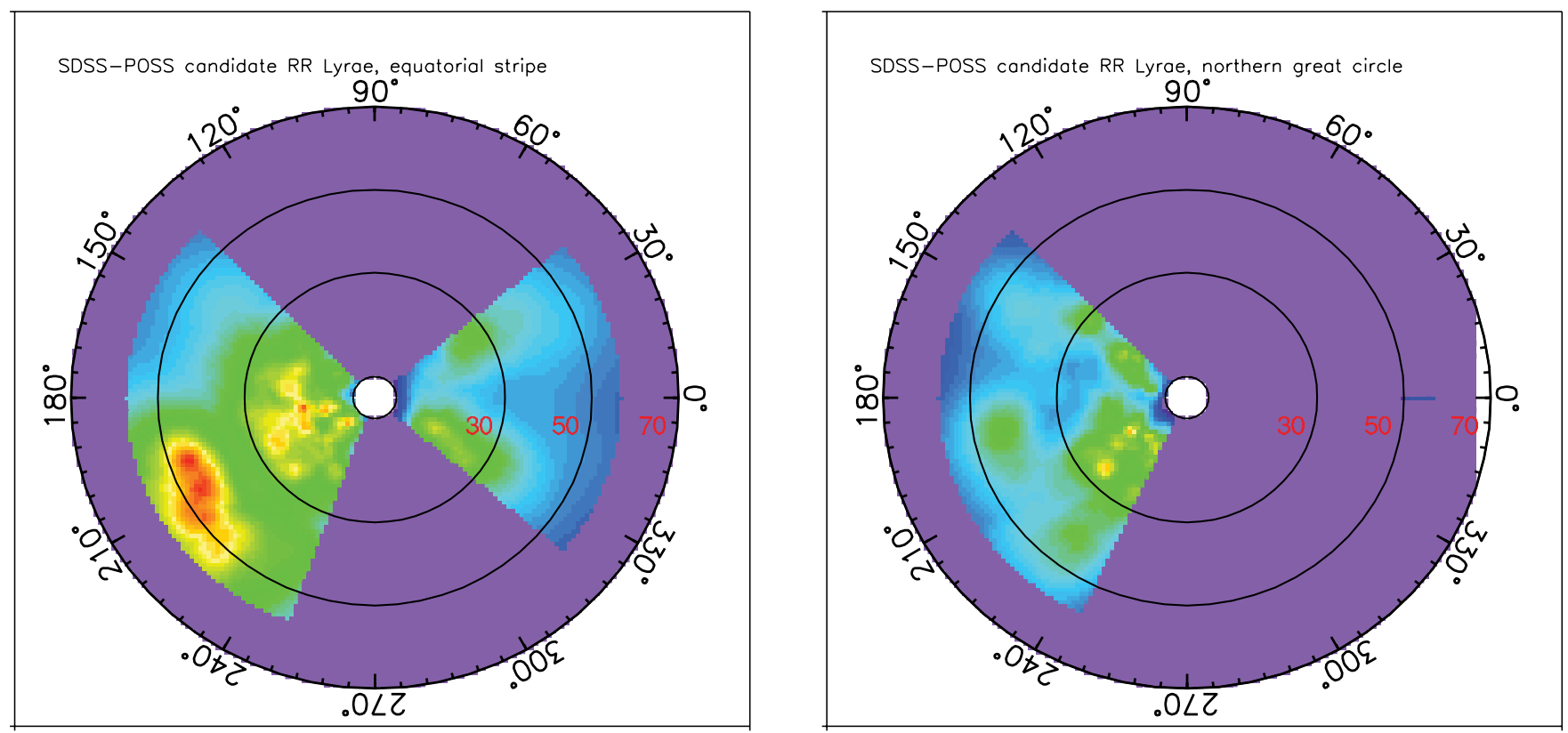

FIG. 23.-Implied volume-density distribution of candidate RR Lyrae stars shown in Fig. 21 (left) and Fig. 22 (right). The figure is designed to ease comparison with Fig. 8 of Ivezić et al. (2005). The color scheme represents the number density multiplied by the cube of the galactocentric radius and displayed on a logarithmic scale with a dynamic range of 300 (light blue to red). The green color corresponds to the mean density; all wedges with the data would have this color if the halo numberdensity distribution followed a perfectly smooth $r^{-3}$ power law. The purple color marks the regions without the data (note that here the maximum probed distance, $57 \mathrm{kpc}$, is smaller than the $70 \mathrm{kpc}$ from Ivezić et al.). The yellow regions are formally of $\sim 3 \sigma$ significance (using only the variance of the counts).

sample size allows us to constrain the overall shape of the $m_{\mathrm{SDSS}}-$ $m_{\text {POSS }}$ distribution and not only its rms scatter, as discussed in $\S 5.1$. The dependence of the quasar variability on rest-frame wavelength and time lag is analyzed in $\S 5.2$.

\subsection{The Distribution of SDSS-POSS Magnitude Differences for Quasars}

Analysis of the multiepoch SDSS imaging data suggests that the distribution of $\Delta m$ for quasars is better described by an exponential distribution than by a Gaussian distribution for all bands (ugriz) and timescales probed (up to a $4 \mathrm{yr}$ time lag in the observer's frame). This result is independent of whether the data are binned by wavelength and time lag in the rest or observer's frame (I04). Here we investigate whether this result can be reproduced for much longer time lags using SDSS-POSS measurements.

Figure 24 shows the magnitude difference distributions for stars and spectroscopically confirmed quasars with redshifts in the range $0.3<z<2.4$, measured using the GSC, DPOSS, and USNO-A2.0 catalogs (we use both GSC and DPOSS catalogs for this analysis because they have different calibrations). The distributions for quasars are marked by triangles, and those for a control sample of stars with the same magnitude distribution ${ }^{21}$ are marked by circles.

The dashed lines in Figure 24 show exponential distributions that have the same rms scatter as the data (the rms values for each distribution are shown in the panels and also listed in Table 5), and the dot-dashed lines show Gaussian distributions, both convolved with a Gaussian of the same width as the distri-

21 The slope of the differential magnitude distribution ("number counts") for quasars is much steeper than that for stars. Since the photometric errors increase with magnitude, care must be taken to properly account for the error contribution to the measurement of the structure function. A simple comparison of two magnitude-limited samples of stars and quasars results in an underestimated photometric error contribution to the structure function. bution of magnitude differences for stars. While the data presented here do not constrain the tails of the $\Delta m$ distributions as well as multiepoch SDSS data do (due to larger photometric errors), the obtained magnitude distributions are consistent with the inferences made using multiepoch SDSS data. Typically, $\sim 1 \%$ of the sample is outside the $\pm 3 \sigma$ boundaries, a fraction about 5 times larger than expected for a Gaussian distribution. While formally significant, it is possible that the remaining calibration problems with POSS catalogs have contributed to this deviation from a perfect Gaussian distribution. In any case, the deviations are sufficiently small for the rms width to be an efficient statistic for describing the observed distributions.

These observed rms values, listed in Table 5, are $\sim 1 \sigma$ ( $\sim 0.05-0.10 \mathrm{mag}$ ) smaller than the values obtained by dV03. The photometric errors (i.e., structure function for stars) displayed in Figure 8 from dV03 correspond to the smaller of the two curves shown in their Figure 4. Adopting the other curve decreases the estimate of the quasar variability as measured by $\mathrm{dV} 03$, and thus decreases the discrepancy with our results to a $<1 \sigma$ level. It is noteworthy that the magnitude difference distributions for stars shown in Figure 24 have smaller rms values than do the structure functions for stars shown in Figure 4 from $\mathrm{dV} 03$ [our values correspond to $\log (\mathrm{SF}) \sim-0.65$ or less]. Thus, it is plausible that the remaining slight discrepancy is due to somewhat different procedures used to recalibrate POSS catalogs. Furthermore, in a follow-up paper, dV05 obtained structure functions for stars (their Fig. 6) that are fully compatible with the $\log (\mathrm{SF}) \sim-0.65$ level obtained here. Hence, both our and the de Vries et al. calibration methods yield essentially the same final photometric accuracy for recalibrated POSS data.

\subsection{The Turnover in the Structure Function}

The extrapolation of the power-law dependence of the quasar rms variability on time, measured on short timescales using repeated SDSS imaging (I04), predicts that the quasar rms variability 

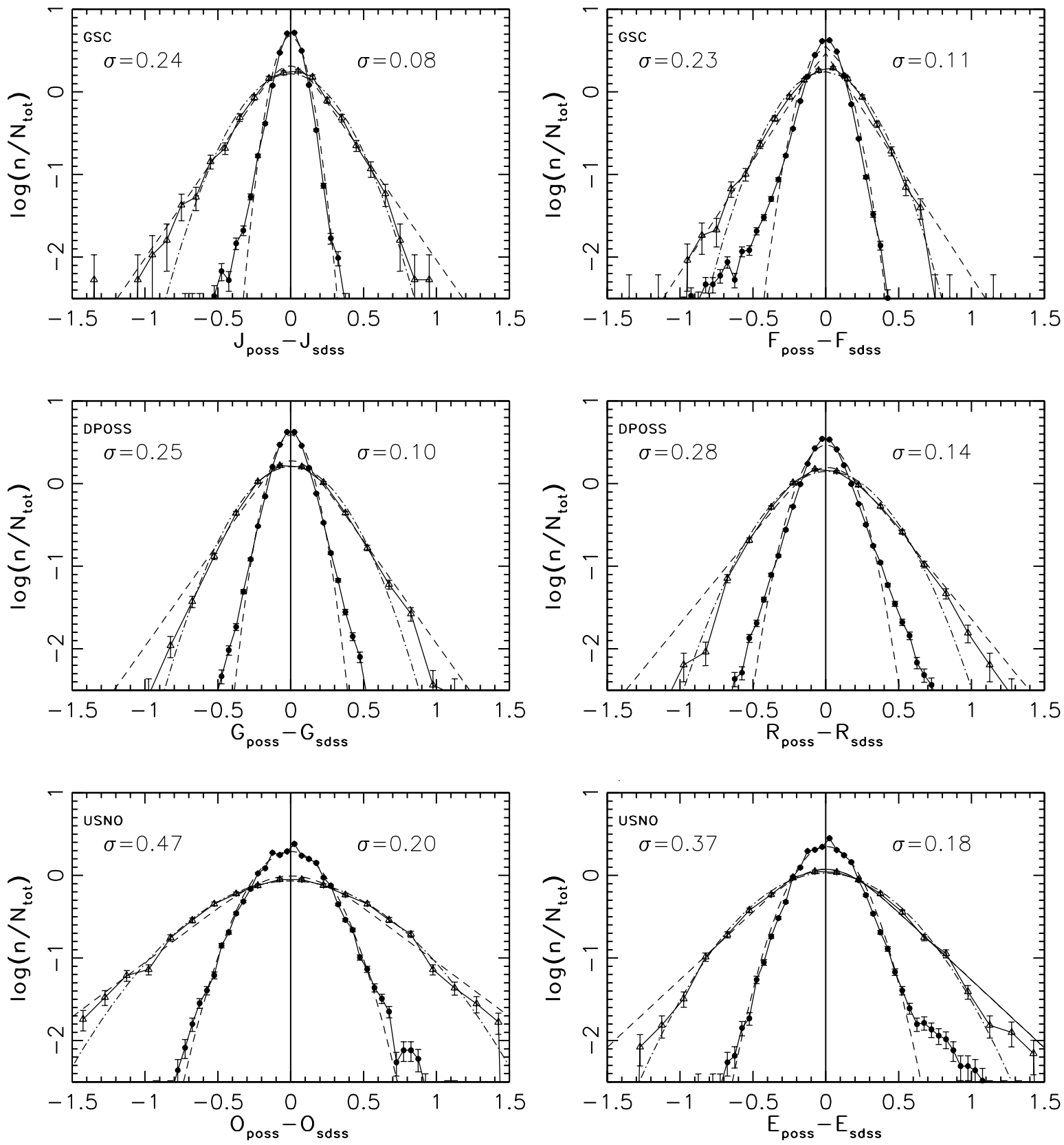

FIG. 24.-Triangles: Probability density distributions of POSS-SDSS magnitude differences for color-selected low-redshift quasars. Circles: Corresponding distribution for stars (note logarithmic scale). The rms for each distribution measured using the interquartile range is also shown in each panel (left value, quasars; right value, stars). The dot-dashed lines show Gaussian distributions that have the same rms scatter as the data. Note that the magnitude differences for the stars are well described by a Gaussian. The dashed lines show exponential distributions that have the same rms as the data for the quasars and are convolved with the error distribution. They seem to provide a marginally better fit to the data than the Gaussian distribution. [See the electronic edition of the Journal for a color version of this figure.]

measured using SDSS and POSS I should be on the order of $0.60 \mathrm{mag}$, and $0.35 \mathrm{mag}$ for SDSS-POSS II. Since the measured values (see Table 5) are smaller than these extrapolated values, they present possible evidence for a turnover in the quasar structure function.

Figure 25 shows the dependence of structure function on rest-frame time lag in the range 2000-3000 $\AA$ for two data sets: SDSS-SDSS for short time lags (taken from I04; note that the variability inferred from repeated imaging scans is fully consistent with the results presented by Vanden Berk et al. [2004], which were based on a comparison of imaging and spectrophotometric magnitudes), and SDSS-POSS for long time lags. The extrapolation of the power law measured for short timescales clearly overestimates the amplitude of the structure func- tion reported here. We fit the observed dependence of the structure function on rest-frame time lag using the following functional form ${ }^{22}$

$$
\mathrm{SF}\left(\Delta t_{\mathrm{RF}}\right)=D\left(1-e^{-\left(\Delta t_{\mathrm{RF}} / \tau\right)^{\gamma}}\right)
$$

The best-fit parameters are $D=0.32 \pm 0.03, \tau=390 \pm 80$ days, and $\gamma=0.55 \pm 0.05$. This best fit is shown in Figure 25 by the dot-dashed line. We conclude that the characteristic timescale

\footnotetext{
${ }^{22}$ This particular form provides a good fit to the data with a small number of free parameters. However, this form may not have any special physical significance.
} 
TABLE 5

The Long-Term Quasar Variability

\begin{tabular}{|c|c|c|c|}
\hline $\begin{array}{l}\text { Band } \\
\text { (1) }\end{array}$ & $\begin{array}{c}\mathrm{rms} \\
(2)\end{array}$ & $\begin{array}{c}\text { Error } \\
\text { (3) }\end{array}$ & $\begin{array}{c}\sigma \\
(4)\end{array}$ \\
\hline J .................................. & 0.24 & 0.08 & 0.23 \\
\hline$F$ & 0.23 & 0.11 & 0.20 \\
\hline$G \ldots \ldots \ldots \ldots \ldots \ldots \ldots \ldots \ldots \ldots \ldots \ldots \ldots$ & 0.25 & 0.10 & 0.23 \\
\hline$R$ & 0.28 & 0.14 & 0.24 \\
\hline$O$ & 0.47 & 0.20 & 0.42 \\
\hline E & 0.37 & 0.18 & 0.32 \\
\hline
\end{tabular}

Notes.-In the observer's frame, "long term" is $\sim 10$ yr for the $J / F$ or $G / R$ bands and $\sim 50 \mathrm{yr}$ for the $O / E$ bands (see Fig. 1). Col. (2): The rms scatter for quasars. Col. (3): The rms scatter for stars with a magnitude distribution similar to that of the selected quasars. Col. (4): An estimate of the intrinsic rms variability of the quasars, $\sigma=\left(\mathrm{rms}^{2}-\text { error }^{2}\right)^{1 / 2}$.

for optical variability of quasars is on the order of 1 yr in the rest frame. This timescale is in good agreement with variability models based on accretion disk instabilities (Kawaguchi et al. 1998; Vanden Berk et al. 2004 and references therein). On the other hand, this conclusion is somewhat different from that derived by $\mathrm{dV} 05$, who analyzed very similar data.

Using a sample of quasars with historic observations from POSS similar to the sample discussed here, dV05 concluded that there is no turnover in the structure function of the quasars (up to timescales of $\sim 40 \mathrm{yr}$ ). Although they used a slightly different time-binning method, a comparison of their Figure 8 (or Fig. 13) and our Figure 25 reveals that the data points have similar behavior. In particular, the point marked "POSS II" in our figure is below the best fit, and this is the same depression visible in Figures 8 and 13 of dV05 (shown by the thin solid line in Fig. 25). Since our two POSS points agree well with the dV05 structure function, the different conclusions are not due to problems with the recalibration of POSS surveys, but rather to differences in the data interpretation.

It is worth pointing out that the asymptotic approach of our best-fit structure function to a value of $0.32 \mathrm{mag}$ is due to the chosen functional form. In a strict sense, POSS I and POSS II points only imply that the power law measured for short timescales (rest-frame time lag $\lesssim 3 \mathrm{yr}$ ) overestimates the amplitude of the structure function at timescales of $\gtrsim 10 \mathrm{yr}$. While the dV05 structure function with its fine time-bin sampling provides some evidence for asymptotic behavior (see Fig. 25), it is entirely possible that the structure function continues to increase with time for long timescales, albeit much more slowly than for short timescales.

Using a model light curve based on repeated outbursts with a given timescale, dV05 concluded that their structure function cannot be explained with a single timescale. By adopting a twotimescale model, they obtained an excellent fit to their structure function, including the depression around the POSS II point in our Figure 25. The discrepancy between our best-fit model curve and the point marked "POSS II" is about $1 \sigma$, or $0.05 \mathrm{mag}$. Given the data and analysis methods presented in this paper, we are not in a position to claim that this is a significant discrepancy. On the other hand, error bars in Figures 8 and 13 from dV05 indicate a much higher statistical significance, probably due to a different analysis method that incorporates finer time-lag sampling than presented here. In principle, some hidden remaining systematic errors at the level of a few hundredths of a magnitude
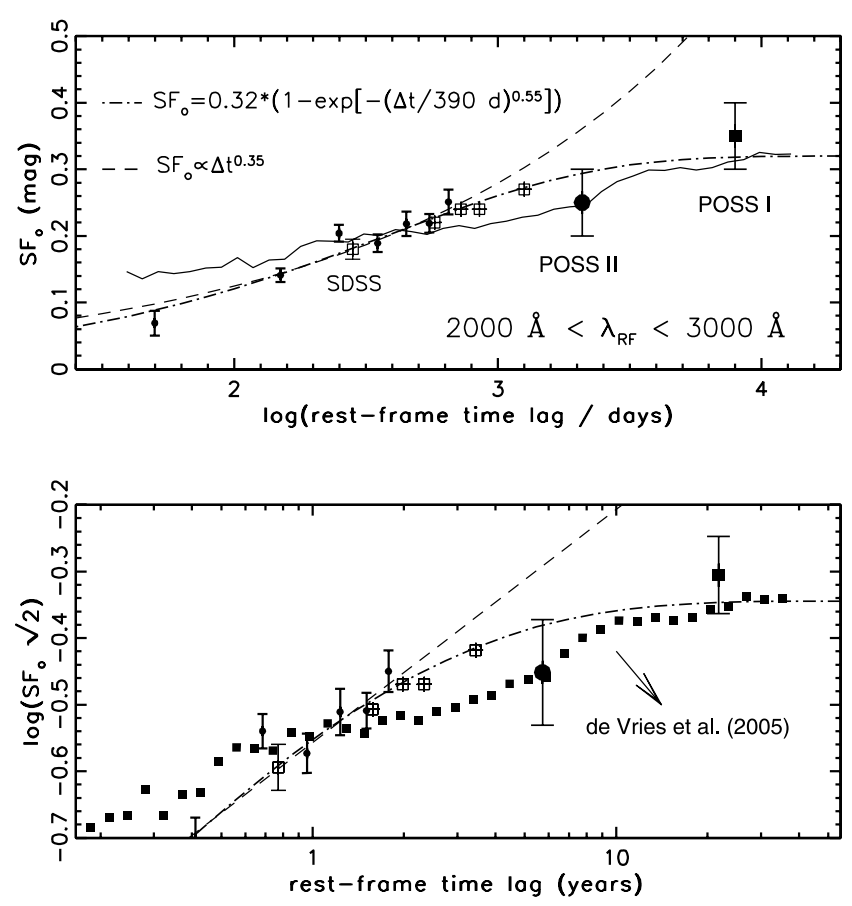

FIG. 25.-Top: Long-term dependence of the structure function on rest-frame time lag, in the range $2000-3000 \AA$, for two data sets: SDSS-SDSS for short time lags (small circles, adopted from I04; open squares, based on the most recent SDSS data), and SDSS-POSS for long time lags (large symbols marked POSS I and POSS II ). The thin solid line is the structure function obtained by dV05. The observed SDSS-POSS long-term variability is smaller than predicted by the extrapolation of the power law measured for short timescales using repeated SDSS imaging (dashed line): the measured values are 0.35 and $0.24 \mathrm{mag}$ (symbols marked POSS I and POSS II, respectively), while the extrapolated values are 0.60 and $0.35 \mathrm{mag}$ for SDSS-POSS I and SDSS-POSS II, respectively. The dot-dashed line shows a simultaneous best fit to SDSS data taken from I04 and displayed by the small circles (eq. [5]). The new SDSS data displayed by open squares were not used in this fit. The bottom panel shows the same data and models, except that the structure function is expressed using the convention from dV05 (e.g., their Figs. 8, 13, and 16), in order to facilitate an easy comparison with their results. [See the electronic edition of the Journal for a color version of this figure.]

cannot be easily excluded, but there is no proof that such errors indeed exist.

One way to resolve this discrepancy is to use more accurate photometric data than recalibrated POSS catalogs. As the SDSS continues, the probed timescales for quasar variability will extend to $\sim 5 \mathrm{yr}$ (in the rest frame) in a $300 \mathrm{deg}^{2}$ region (the socalled Southern Survey and the SDSS II supernova survey; Sako et al. 2005). Hence, it will soon be possible to measure the quasar structure function, and to test for the existence of multiple timescales, using only SDSS data. At the time of writing, most of the recent SDSS data are still not properly photometrically calibrated (because most of the new scans were obtained in nonphotometric conditions). However, a subset of data from the Southern Survey area is already calibrated and can be used to extend results from I04 to rest-frame timescales of up to $3.5 \mathrm{yr}$ for a sample of 3000 spectroscopically confirmed SDSS quasars. The structure function based on this preliminary analysis is shown by open squares in Figure 25. Although these data were not used to constrain the best-fit empirical model (eq. [5]), the additional points are consistent with it and suggest that the depression in the structure function implied by POSS II data may not be real.

If this depression is not real, then the best-fit parameters from dV05 will have to be revised. However, such a revision would not necessarily imply that their conclusion about multiple timescales 
is incorrect. Within the context of their adopted light-curve model, it is not obvious that our best-fit structure function (which does not include a depression) could be explained by a single timescale. Indeed, our inability to fit the data with $\gamma \sim 1$ (see eq. [5]) may be a strong argument in favor of multiple scales. A useful exercise would be to attempt to explain the best-fit structure function presented here with the light-curve model from $\mathrm{dV} 05$, which is beyond the scope of this work. Nevertheless, a characteristic timescale ( $\tau$ from eq. [5]), which in some sense is an average over the quasar population and possible multiple timescales, appears to be on the order of $1 \mathrm{yr}$.

\section{DISCUSSION}

We have presented a direct comparison of photometric measurements available in public POSS catalogs. The most accurate photometry is provided by the GSC 2.2 catalog. The results of photometric recalibration based on a dense grid of calibration stars measured by the SDSS demonstrate that errors in POSS photometry can be reduced by about a factor of 2 . POSS I magnitudes can be brought to $\sim 0.15$ mag accuracy, and POSS II magnitudes to $\sim 0.10$ mag accuracy. While these apparently irreducible errors are considerably larger than those delivered by modern CCD data $(\sim 0.02)$, the POSS catalogs are, nevertheless, invaluable for studying sources variable on long timescales. A particular success of the recalibration method is that the resulting error distribution for POSS photometry is nearly Gaussian, which greatly helps in the design of robust algorithms for selecting candidate variable sources.

We have designed and tested algorithms for selecting candidate variable sources using POSS and SDSS photometric measurements. The algorithm's decision to tag a source as a candidate variable is correct in more than $75 \%$ of cases, and approaches $95 \%$ for sources outside the stellar locus.

This is the first study to examine the distribution of sources variable on long timescales in SDSS color-color diagrams. Even with the fairly large cutoffs for selecting candidate variables $(0.20-0.35 \mathrm{mag})$, we find that at least $1 \%$ of faint optical sources appear variable.

A particularly valuable result of comparing POSS and SDSS catalogs is the selection of candidate RR Lyrae stars, which are excellent probes of the Milky Way's halo structure. We have demonstrated that the known halo substructures are recovered by the selected candidates and discovered several new features. This method improves the efficiency of color-based selection proposed by Ivezić et al. (2005) and will eventually yield several thousand highly reliable RR Lyrae candidates. A simultaneous analysis of samples selected by different methods will provide a good assessment of their contamination and completeness rates.

About $10 \%$ of the variable population are quasars, although they represent only $0.25 \%$ of all point sources (for $g<19$ ). Using a sample of $\sim 17,000$ spectroscopically confirmed quasars, we have demonstrated that the power-law increase of the quasar variability (structure function, rms) with time lag observed for short time lags cannot be extrapolated beyond a few years in the rest frame; such extrapolation predicts a variability level significantly larger than measured ( 0.35 vs. 0.60 mag for SDSSPOSS I, and 0.24 vs. 0.35 mag for SDSS-POSS II). The implied turnover in structure function indicates that the characteristic timescale for optical variability of quasars is on the order $1 \mathrm{yr}$ in the rest frame. This timescale is in good agreement with variability models based on accretion disk instabilities. However, a detailed comparison with competing models, such as supernova bursts and microlensing (see Vanden Berk et al. 2004 and references therein) is necessary before more robust conclusions can be reached.
Our study has also revealed some limitations of the POSS catalogs. In particular, we had to limit our search for variable sources to only isolated point sources detected by both POSS and SDSS. Attempts to find sources detected by only one survey, or variable sources that are blended with another nearby source, were unsuccessful due to overwhelmingly large numbers of false positives.

Despite these shortcomings, the assembled catalogs of candidate variable sources offer a good starting point for further analysis and follow-up observations. For example, light curves and spectra for selected candidates could be obtained even with telescopes of modest size. Such additional data would help improve candidates' classification beyond information provided by SDSS colors. Another potentially interesting research direction is positional cross-correlation with catalogs obtained at other wavelengths (e.g., ROSAT, 2MASS, IRAS). For example, long-period variables such as Mira and other asymptotic giant branch stars are typically strong infrared emitters, and thus could be efficiently separated from the rest of the candidate variables. In order to facilitate such studies, we have made our catalog of recalibrated POSS photometric observations publicly available (see Appendix B).

This study once again demonstrates the importance of maintaining a careful archive of astronomical observations; the data may be valuable long after the acquisition technology becomes obsolete.

We thank Princeton University for generous financial support of this research. We are grateful to Wim de Vries for making results from de Vries et al. (2005) available in machine-readable format and for illuminating discussions.

Funding for the creation and distribution of the SDSS Archive has been provided by the Alfred P. Sloan Foundation, the Participating Institutions, the National Aeronautics and Space Administration, the National Science Foundation, the US Department of Energy, the Japanese Monbukagakusho, and the Max Planck Society. The SDSS Web site is http://www.sdss.org.

The SDSS is managed by the Astrophysical Research Consortium for the Participating Institutions. The Participating Institutions are the University of Chicago, Fermilab, the Institute for Advanced Study, the Japan Participation Group, the Johns Hopkins University, Los Alamos National Laboratory, the Max Planck Institute for Astronomy, the Max Planck Institute for Astrophysics, New Mexico State University, the University of Pittsburgh, Princeton University, the United States Naval Observatory, and the University of Washington.

\section{APPENDIX A}

\section{AN OVERVIEW OF POSS CATALOGS USED IN THIS WORK}

USNO catalogs.-USNO-A2.0 is a catalog of 526,280,881 stars, based on a rereduction of the Precision Measuring Machine scans of the POSS I (Minkowski \& Abel 1963) $O$ and $E$ plates, the UK Science Research Council SRC-J survey plates, and the European Southern Observatory ESO-R survey plates. For field centers with $\delta>-30^{\circ}$, data come from the POSS I plates, while data for field centers with $\delta<-35^{\circ}$ come from the SRC-J and ESO-R plates. The USNO-A2.0 catalog uses the International Celestial Reference Frame as realized by the USNO ACT catalog (Urban et al. 1997), and in addition to source 
coordinates, it lists the blue $(O)$ and red $(E)$ magnitudes for each object. The USNO-B catalog (Monet et al. 2003), currently released in version 1.0, is the next in a sequence of catalogs produced $^{23}$ by the USNO team. It is an all-sky catalog with positions, proper motions, magnitudes in different optical bands $(O, E, J, F$, $N)$, and star/nonstar estimations for approximately 1 billion objects. Besides the first-epoch surveys (POSS I, ESO-R, SRC-J), it also utilizes the second-epoch surveys: POSS II for field centers with $\delta>-30^{\circ}$, and SES (South Equatorial Survey) for $\delta<$ $-35^{\circ}$ (Reid et al. 1991). It is fairly complete to $V=21$, with a claimed astrometric accuracy of 0 "'2 (J2000.0), a photometric accuracy of $0.3 \mathrm{mag}$, and an $85 \%$ accuracy for distinguishing stars from nonstellar objects.

The Guide Star Catalog.-The GSC II (McLean et al. 2000) is an all-sky catalog based on scans of the photographic plates obtained by the Palomar and UK Schmidt telescopes. Schmidt plates for both the northern (POSS II) and southern (SES) hemisphere surveys were digitized on the GAMMA scanning machines. Positions, magnitudes, and classifications are produced for all objects on each plate, and the data are stored in the COMPASS database. The GSC2.2 catalog is an all-sky, magnitude-selected export of calibrated source parameters from the COMPASS database, complete to $F=18.5 \mathrm{mag}$ and $J=19.5 \mathrm{mag}$. It uses $\sim 1000$ objects per plate for astrometric calibration, resulting in astrometric errors of 0.3 , and $\sim 100$ objects per plate for photometric calibration, resulting in errors in the range $0.2-$ $0.25 \mathrm{mag}$. The number of unique objects exported is approximately 456 million. $^{24}$

The Digitized Second Palomar Observatory Sky Survey.DPOSS (Djorgovski et al. 1998) is a digitized version of POSS II, based on plate scans done at the Space Telescope Science Institute, CCD calibrations done at Palomar, and processing done

\footnotetext{
23 Available from http://www.nofs.navy.mil.

${ }^{24}$ For more details and access to catalogs, see http://www-gsss.stsci.edu/ Catalogs/GSC/GSC2/GSC2.htm.
}

at Caltech. DPOSS consists of the original image database ( $\sim 3$ TB of pixel data) and the derived catalogs and metadata, primarily the Palomar-Norris Sky Catalog. DPOSS is a survey of the northern sky $\left(\delta>-3^{\circ}\right)$ in three bands (photographic $J, F$, and $N$, calibrated to $g, r$, and $i$; note that we use uppercase letters for DPOSS magnitudes to distinguish them from SDSS magnitudes), with typical limiting magnitudes $G \sim 21-21.5$, $R \sim 21$, and $I \sim 19.5$ mag. Accurate star-galaxy classification is available for all objects to $\sim 1-1.5$ mag above the detection limit. $^{25}$ The initial data release covers the high Galactic latitudes. The final catalog is expected to contain about 50 million galaxies and a billion stars.

\section{APPENDIX B}

\section{THE SDSS-POSS CATALOG OF RECALIBRATED OBSERVATIONS}

In order to facilitate follow-up studies of variable objects described in this work, we have made the catalog of recalibrated POSS photometric observations publicly available at our Web site. $^{26}$ The catalog provides SDSS and POSS data for $6,811,791$ point sources from 84 SDSS observing runs obtained before 2002 July 1 and covers an area of roughly $3300 \mathrm{deg}^{2}$ (SDSS Data Release 2). This site also includes a detailed description of the catalog, which lists SDSS astrometry, photometric measurements (five SDSS magnitudes and their errors, and various photometric flags), and the original and recalibrated USNO-B ( $O$ and $E$ bands) and DPOSS ( $G, R$, and $I$ bands) magnitudes with corresponding error estimates. The samples and analysis described in this paper can be fully reproduced using these data.

\footnotetext{
25 The survey and selected data products are publicly available from http:// astro.ncsa.uiuc.edu/catalogs/dposs.

${ }^{26}$ See http://www.astro.washington.edu/bsesar/sdss-poss-cro.
}

Abazajian, K., et al. 2004, AJ, 128, 502

Akerlof, C., et al. 2000, AJ, 119, 1901

Cid Fernandes, R., Sodré, L., Jr., \& Vieira da Silva, L., Jr. 2000, ApJ, 544, 123 de Vries, W. H., Becker, R. H., \& White, R. L. 2003, AJ, 126, 1217 (dV03)

de Vries, W. H., Becker, R. H., White, R. L., \& Loomis, C. 2005, AJ, 129, 615 (dV05)

Djorgovski, S. G., Gal, R. R., Odenwahn, S. C., de Carvalho, R. R., Brunner, R., Longo, G., \& Scaramella, R. 1998, in Wide Field Surveys in Cosmology, ed. S. Colombi, Y. Mellier, \& B. Raban (Paris: Editions Frontières), 89

Eyer, L. 1999, Baltic Astron., 8, 321

Fan, X. 1999, AJ, 117, 2528

Finlator, K., et al. 2000, AJ, 120, 2615

Fukugita, M., Ichikawa, T., Gunn, J. E., Doi, M., Shimasaku, K., \& Schneider, D. P. 1996, AJ, 111,1748

Groot, P. J., et al. 2003, MNRAS, 339, 427

Gunn, J. E., et al. 1998, AJ, 116, 3040

Hawkins, M. R. S. 1983, MNRAS, 202, 571

Hawkins, M. R. S., \& Véron, P. 1995, MNRAS, 275, 1102

Hawley, S. L., et al. 2002, AJ, 123, 3409

Helmi, A., et al. 2003, ApJ, 586, 195

Hogg, D. W., Finkbeiner, D. P., Schlegel, D. J., \& Gunn, J. E. 2001, AJ, 122, 2129

Ivezić, Ž., Vivas, A. K., Lupton, R. H., \& Zinn, R. 2005, AJ, 129, 1096

Ivezić, Ž., et al. 2000, AJ, 120, 963

2001, AJ, 122, 2749

2003, Mem. Soc. Astron. Italiana, 74, 978

2004a, Astron. Nachr., 325, 583

2004b, in IAU Symp. 222, The Interplay among Black Holes, Stars, and ISM in Galactic Nuclei, ed. T. Storchi-Bergmann, L. C. Ho, \& H. R. Schmitt (Cambridge: Cambridge Univ. Press), 525 (I04)

\section{REFERENCES}

Ivezić, Ž., et al. 2004c, in ASP Conf. Ser. 317, Milky Way Surveys: The Structure and Evolution of Our Galaxy, ed. D. Clemens, R. Shah, \& T. Brainerd (San Francisco: ASP), 179

2004d, in ASP Conf. Ser. 327, Satellites and Tidal Streams, ed. F. Prada, D. Martinez Delgado, \& T. J. Mahoney (San Francisco: ASP), 104

Kaiser, N., et al. 2002, Proc. SPIE, 4836, 154

Kawaguchi, T., Mineshige, S., Umemura, M., \& Turner, E. L. 1998, ApJ, 504, 671

Lattanzi, M. G., \& Bucciarelli, B. 1991, A\&A, 250, 565

Lenz, D. D., Newberg, J., Rosner, R., Richards, G. T., \& Stoughton, C. 1998, ApJS, 119, 121

Lupton, R. H., Ivezić, Ž., Gunn, J. E., Knapp, G. R., Strauss, M. A., \& Yasuda, N. 2002, Proc. SPIE, 4836, 350

Martini, P., \& Schneider, D. P. 2003, ApJ, 597, L109

Matthews, T. A., \& Sandage, A. R. 1963, ApJ, 138, 30

McLean, B. J., Greene, G. R., Lattanzi, M. G., \& Pirenne, B. 2000, in ASP Conf. Ser. 216, Astronomical Data Analysis Software and Systems IX, ed. N. Manset, C. Veillet, \& D. Crabtree (San Francisco: ASP), 145

Minkowski, R., \& Abell, G. O. 1963, in Stars and Stellar Systems, Vol. 3, Basic Astronomical Data, ed. K. A. Strand (Chicago: Univ. Chicago Press), 481

Monet, D. G., et al. 2003, AJ, 125, 984

Munn, J. A., et al. 2004, AJ, 127, 3034

Oke, J. B., \& Gunn, J. E. 1983, ApJ, 266, 713

Paczyński, B. 1997, in Variables Stars and the Astrophysical Returns of the Microlensing Surveys, ed. R. Ferlet, J.-P. Maillard, \& B. Raban (Paris: Editions Frontières), 357 2000, PASP, 112, 1281

2001, in Mining the Sky, ed. A. J. Banday, S. Zaroubi, \& M. Bartelmann (Heidelberg: Springer)

Pier, J. R., Munn, J. A., Hindsley, R. B., Hennesy, G. S., Kent, S. M., Lupton, R. H., \& Ivezić, Ž. 2003, AJ, 125, 1559 
Reid, I. N., et al. 1991, PASP, 103, 661

Richards, G. T., et al. 2002, AJ, 123, 2945

Sako, M., et al. 2005, preprint (astro-ph/0504455)

Schlegel, D., Finkbeiner, D. P., \& Davis, M. 1998, ApJ, 500, 525

Schneider, D. P., et al. 2003, AJ, 126, 2579

Smith, J. A., et al. 2002, AJ, 123, 2121

Stoughton, C., et al. 2002, AJ, 123, 485

Trevese, D., Kron, R. G., \& Bunone, A. 2001, ApJ, 551, 103

Tyson, J. A. 2002, Proc. SPIE, 4836, 10

Udalski, A., et al. 2002, Acta Astron., 52, 115
Urban, S. E., Corbin, T. E., \& Wycoff, G. L. 1997, ACT Reference Catalog (Washington: USNO)

van den Bergh, S., Herbst, E., \& Pritchet, C. 1973, AJ, 78, 375

Vanden Berk, D. E., et al. 2004, ApJ, 601, 692

Vestrand, W. T., et al. 2002, Proc. SPIE, 4845, 126

Vivas, A. K., et al. 2001, ApJ, 554, L33

Woźniak, P. R., et al. 2002, Acta Astron., 52, 129

Yanny, B., et al. 2000, ApJ, 540, 825

York, D. G., et al. 2000, AJ, 120, 1579 